Review

\title{
Environmental Factors That Affect Parathyroid Hormone and Calcitonin Levels
}

\author{
Mirjana Babić Leko (D), Nikolina Pleić (1), Ivana Gunjača (1) and Tatijana Zemunik *(1) \\ Department of Medical Biology, School of Medicine, University of Split, Šoltanska 2, 21000 Split, Croatia; \\ mbabic@mefst.hr (M.B.L.); npleic@mefst.hr (N.P.); igunjaca@mefst.hr (I.G.) \\ * Correspondence: tzemunik@mefst.hr; Tel.: +385-2155-7888
}

check for updates

Citation: Babić Leko, M.; Pleić, N.; Gunjača, I.; Zemunik, T.

Environmental Factors That Affect Parathyroid Hormone and Calcitonin Levels. Int. J. Mol. Sci. 2022, 23, 44. https://doi.org/10.3390/ijms23010044

Academic Editors: Concetta

Ambrosino and Alberto Falchetti

Received: 15 November 2021

Accepted: 19 December 2021

Published: 21 December 2021

Publisher's Note: MDPI stays neutral with regard to jurisdictional claims in published maps and institutional affiliations.

Copyright: (C) 2021 by the authors. Licensee MDPI, Basel, Switzerland. This article is an open access article distributed under the terms and conditions of the Creative Commons Attribution (CC BY) license (https:// creativecommons.org/licenses/by/ $4.0 /)$.

\begin{abstract}
Calciotropic hormones, parathyroid hormone (PTH) and calcitonin are involved in the regulation of bone mineral metabolism and maintenance of calcium and phosphate homeostasis in the body. Therefore, an understanding of environmental and genetic factors influencing PTH and calcitonin levels is crucial. Genetic factors are estimated to account for $60 \%$ of variations in PTH levels, while the genetic background of interindividual calcitonin variations has not yet been studied. In this review, we analyzed the literature discussing the influence of environmental factors (lifestyle factors and pollutants) on PTH and calcitonin levels. Among lifestyle factors, smoking, body mass index (BMI), diet, alcohol, and exercise were analyzed; among pollutants, heavy metals and chemicals were analyzed. Lifestyle factors that showed the clearest association with PTH levels were smoking, BMI, exercise, and micronutrients taken from the diet (vitamin D and calcium). Smoking, vitamin D, and calcium intake led to a decrease in PTH levels, while higher BMI and exercise led to an increase in PTH levels. In terms of pollutants, exposure to cadmium led to a decrease in PTH levels, while exposure to lead increased PTH levels. Several studies have investigated the effect of chemicals on PTH levels in humans. Compared to PTH studies, a smaller number of studies analyzed the influence of environmental factors on calcitonin levels, which gives great variability in results. Only a few studies have analyzed the influence of pollutants on calcitonin levels in humans. The lifestyle factor with the clearest relationship with calcitonin was smoking (smokers had increased calcitonin levels). Given the importance of PTH and calcitonin in maintaining calcium and phosphate homeostasis and bone mineral metabolism, additional studies on the influence of environmental factors that could affect PTH and calcitonin levels are crucial.
\end{abstract}

Keywords: environmental factors; PTH; calcitonin; pollutants; lifestyle factors; calcium; phosphate; vitamin D

\section{Introduction}

Maintenance of calcium homeostasis in the body is crucial since calcium regulates various physiological processes, including cellular signaling, protein and enzyme function, neurotransmission, contractility of the muscles, and blood coagulation [1]. Calcium homeostasis is regulated by parathyroid hormone (PTH), calcitonin, the active form of vitamin $\mathrm{D}(1 \alpha, 25$-dihydroxyvitamin $\mathrm{D}(1,25(\mathrm{OH}) 2 \mathrm{D} 3))$, and serum calcium and phosphate levels. Regulation of phosphate metabolism is also important as phosphate is involved in protein and enzyme function, cell signaling, and skeletal mineralization and is a component of cell membranes and nucleic acids [2,3]. The main factors that regulate phosphate homeostasis are PTH, fibroblast growth factor 23 (FGF-23), 1,25(OH)2D3, and Klotho [3]. Calcitonin is also involved in the regulation of phosphate levels [4,5]. PTH is released from the parathyroid glands [6], while calcitonin is released from thyroid C-cells [7]. Alternation of PTH levels can lead to the development of hyperparathyroidism and hypoparathyroidism. Changes in calcitonin levels have also been observed in pathological conditions (such as medullary thyroid carcinoma [8]). Therefore, variations in PTH and calcitonin levels may indicate that the normal functioning of parathyroid glands and thyroid is altered. Various 
factors can affect PTH and calcitonin levels, such as genetic factors [9-11], demographic factors (age [12-14], sex [15-17]), and environmental factors [18-21]. It is estimated that genetic factors account for $60 \%$ of variations in PTH levels [9], while the amount to which genetic factors contribute to interindividual variation in calcitonin levels has not been studied. This review aims to provide an insight into environmental factors (lifestyle factors and pollutants) that affect PTH and calcitonin levels (Figure 1).

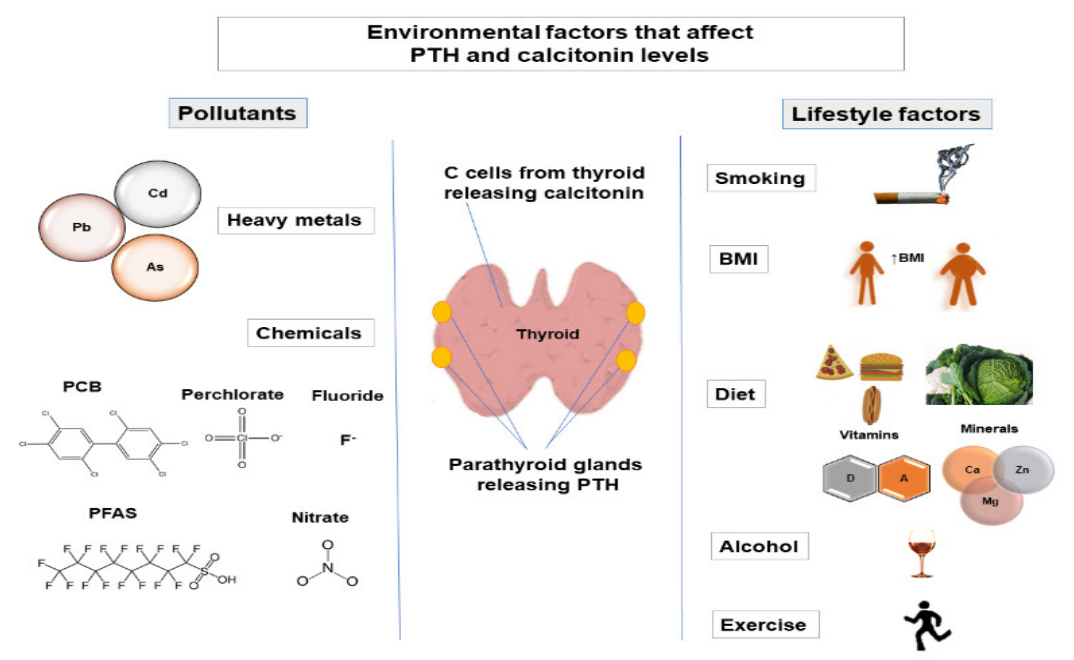

Figure 1. Environmental factors (lifestyle factors and pollutants) that affect PTH and calcitonin levels. As, arsenic; BMI, body mass index; Ca, calcium; $\mathrm{Cd}$, cadmium; F, fluoride; $\mathrm{Mg}$, magnesium; $\mathrm{Pb}$, lead; PCB, polychlorinated biphenyl; PFAS, perfluoroalkyl substances; PTH, parathyroid hormone; Zn, zinc.

\section{Involvement of PTH and Calcitonin in the Regulation of Calcium and Phosphate Levels}

Calcium and phosphate levels in the body are regulated by the complex intestinebone-kidney-parathyroid axis [22]. Calcium homeostasis is regulated by PTH, calcitonin, $1,25(\mathrm{OH}) 2 \mathrm{D} 3$, and serum phosphate and calcium levels. PTH increases calcium levels in the body, and calcitonin decreases calcium levels in the body. PTH increases serum calcium levels by activating osteoclasts (cells involved in bone resorption) and absorbing calcium in the kidneys. Calcitonin lowers calcium levels by inhibiting osteoclasts [23]. Additionally, 1,25(OH)2D3 stimulates intestinal calcium absorption [24]. Increasing serum levels of 1,25(OH)2D3 and calcium decrease PTH secretion, while increasing serum phosphate levels increase PTH secretion [25]. In addition to PTH, phosphate levels are mainly regulated by FGF-23, 1,25(OH)2D3, Klotho, and dietary phosphate [3,22,26,27], while calcitonin also affects phosphate levels [4,5]. PTH, FGF-23, and Klotho decrease serum phosphate levels (by inhibiting renal phosphate reabsorption), while 1,25(OH)2D3 increases serum phosphate levels (by increasing renal phosphate reabsorption, phosphate absorption from the intestine, and phosphate release from the bones) [2,22]. It has been suggested that FGF-23 acts in a negative feedback loop with PTH [28]; PTH stimulates FGF-23 production [28], while FGF-23 has been shown to inhibit PTH secretion indirectly (by increasing urinary phosphate excretion) and directly (by acting directly on parathyroid glands) [29]. Additionally, a negative feedback mechanism was observed between FGF-23 and 1,25(OH)2D3; 1,25(OH)2D3 increases FGF-23 levels, and FGF-23 decreases 1,25(OH)2D3 levels (by suppressing the expression of $1 \alpha$-hydroxylase-the enzyme responsible for the production of $1,25(\mathrm{OH}) 2 \mathrm{D} 3)$ (reviewed in [22]).

\section{Environmental Factors That Affect PTH and Calcitonin Levels}

\subsection{Lifestyle Factors}

\subsubsection{Smoking}

Many studies have investigated the impact of smoking on PTH levels. Most of these studies reported a decrease in PTH levels in smokers (Table 1). The three largest studies that 
involved more than 7000 participants confirmed these results [30-32]. The study of DiazGomez et al., even showed that maternal smoking decreases PTH levels in newborns [33]. The heavy metal cadmium and thiocyanate (that is converted from cyanide in tobacco) which are also toxic components of tobacco smoke have been shown to reduce PTH levels [19,34]. Jorde et al., observed that after smoking cessation, PTH levels return to normal [30]. The mechanism by which smoking affects PTH levels is not fully understood. PTH-vitamin D axis dysfunction has been observed in smokers [35]. Many studies have found a decrease in 1,25(OH)2D levels among smokers (reviewed in [36]). Although under physiological conditions, a decrease in 1,25(OH)2D levels was accompanied by an increase in PTH levels, this was not observed in smokers in most studies. Need et al., suggested that smoking impairs osteoblast function, increasing serum calcium, which in turn leads to a decrease in PTH levels [37]. Jorde et al. did not rule out a possible direct toxic effect of smoking on parathyroid cells [30]. Additionally, it has been suggested that a decrease in bone mineral density (BMD) among smokers [38] may contribute to PTH-vitamin D axis dysfunction [35].

Most studies investigating the effect of smoking on calcitonin levels have found an increase in calcitonin levels in smokers (Table 2). A large population study by Song et al., involving 10,566 participants showed an increase in calcitonin levels in male smokers [17]. Smoking affects the normal functioning of the thyroid gland [39]; however, the effect of smoking on calcitonin-producing $C$ cells has not been elucidated [17]. The results of Tabassian et al. suggested that the lungs are the source of increased calcitonin in smokers rather than the thyroid. Specifically, smoking increases the release of calcitonin from neuroendocrine lung cells [40].

\subsubsection{Body Mass Index}

Many studies have investigated the influence of body mass index (BMI) on PTH levels. Most studies have shown that an increase in BMI is accompanied by an increase in PTH levels (Table 1). However, a study by Yuan et al., showed a positive correlation between BMI and PTH levels in subjects with lower PTH levels (below $65.8 \mathrm{pg} / \mathrm{mL}$ ), while a negative correlation was observed between BMI and PTH levels in the group of patients with high PTH levels (above $147 \mathrm{pg} / \mathrm{mL}$ ) [41]. There are several possible explanations for the positive correlation between BMI and PTH levels. The first possibility is that weight gain leads to an increase in PTH levels by sequestration of 25-hydroxyvitamin $\mathrm{D}(25(\mathrm{OH}) \mathrm{D})$ in adipose tissue (since $25(\mathrm{OH}) \mathrm{D}$ is soluble in fat) $[42,43]$. Because PTH and $25(\mathrm{OH}) \mathrm{D}$ are inversely related, a decrease in 25(OH)D levels increases PTH levels. Another possibility is that an increase in PTH levels causes weight gain. Because PTH can activate $1 \alpha$-hydroxylase (the enzyme responsible for the production of 1,25(OH)2D), an increase in PTH levels can lead to an increase in 1,25(OH)2D levels. Both PTH and 1,25(OH)2D increase calcium levels. Increased calcium levels in adipocytes result in increased lipid storage (by activation of phosphodiesterase $3 \beta$ which reduces catecholamine-induced lipolysis [44,45]). A possible explanation of the negative correlation between PTH and BMI in patients with high PTH levels is that PTH in higher concentrations inhibits adipogenesis, consequently resulting in weight loss [46]. Additionally, high-dose PTH has been shown to increase the expression of thermogenesis genes, resulting in white adipose browning [47].

Several studies have investigated the association between BMI and calcitonin levels, reporting conflicting results (Table 2). The largest study, which included 9340 people with type 2 diabetes, showed a positive correlation between BMI and calcitonin levels [48]. However, a study by Song et al., conducted on 4638 healthy individuals did not show an association between BMI and calcitonin [17]. Although the relationship between calcitonin levels and BMI in humans has not been fully elucidated, experimental studies have shown that salmon calcitonin intake causes weight loss (reviewed in [49]). These authors also described some additional compounds that target the calcitonin receptor and that could be used as an option in the treatment of obesity [49]. 


\subsubsection{Diet}

Different types of food can affect the level of PTH in the body (Table 1). A diet high in phosphorus and low in calcium has been shown to increase PTH levels [50,51]. This is logical because both high serum phosphate levels and low serum calcium levels are signals to increase PTH release [52]. Phosphorus is present in various types of food and food additives, while dairy products contain a large amount of calcium. Increased intake of dairy products and decreased intake of highly processed food should increase calcium levels and reduce phosphorus levels [51]. Processed foods such as sausages, salami, and white bread [21] and a proinflammatory diet (processed and red meat, refined carbohydrates, and fried food) [53] have been observed to increase PTH levels. Consumption of this type of food increases BMI, which is positively correlated with PTH levels (Table 1). A decrease in PTH levels was observed in consumers of bran bread [21]. A low-protein diet was associated with an increase in PTH levels [54-56]. Interestingly, the consumption of plant foods also led to an increase in PTH levels [21,57]. Therefore, vegans [58] and vegetarians [59] had higher levels of PTH than controls. A possible explanation for this is that higher plant food intake increases serum phosphorus levels (due to pesticide treatment of plants) [60]. PTH levels either decreased $[30,61]$ or did not change $[32,62,63]$ after coffee consumption.

The effect of different types of food on calcitonin levels has not been studied to date. Several studies have shown that food intake (without specifying the type of food) does not affect calcitonin levels $[64,65]$. Zayed et al., have shown that calcitonin levels increase after ingestion of food (without specifying the type of food) [66]. A study in pigs showed that a diet high in phosphorus increased calcitonin levels [67], while a study in rats showed that a diet high in fat increased calcitonin levels [68].

\section{Micronutrients}

Many studies have tested the effect of vitamin D on PTH levels because these two hormones act together. About $95 \%$ of vitamin D is synthesized in the skin after exposure to sunlight, while 5\% of vitamin D comes from food [69]. Since PTH and the active form of vitamin $\mathrm{D}(1,25(\mathrm{OH}) 2 \mathrm{D})$ are in an inverse relationship, it is not surprising that most of the studies have reported a decrease in PTH levels after vitamin D intake (Table 1). In some studies, however, there was no change in PTH levels after vitamin D intake (Table 1). On the other hand, a meta-analysis by Moslehi et al. confirmed that PTH levels are reduced by vitamin D intake [70]. Vitamin A intake decreased [63,71] or did not affect PTH levels [72]. In vitro studies in human [73] and bovine parathyroid cells [74] have shown that retinoic acid (a metabolite of vitamin A) directly suppresses PTH secretion.

No changes in calcitonin levels were observed after vitamin D intake [75]. While calcitonin stimulates $1,25(\mathrm{OH}) 2 \mathrm{D}$ synthesis, $1,25(\mathrm{OH}) 2 \mathrm{D}$ reduces the synthesis of calcitonin [76]. Therefore, it is necessary to conduct additional studies on the relationship between vitamin $\mathrm{D}$ and calcitonin.

Most studies have shown that calcium intake decreases PTH levels (Table 1), which is logical since PTH is released in hypocalcemia. Magnesium intake either increased [77,78] or did not affect [32,79] PTH levels. The relationship between PTH and magnesium is complex because PTH improves magnesium absorption [80], and magnesium reduces PTH secretion in a state of moderately low calcium concentration [81,82]. Zinc intake [83] did not affect PTH levels. However, a study in rats showed that a zinc-deficient diet increased PTH levels [84], while patients with primary hyperparathyroidism had decreased serum zinc levels [85].

Zinc intake decreased calcitonin levels [83,86], while copper intake [86] did not affect calcitonin levels. Intake of both zinc and copper resulted in inhibition of bone loss $[87,88]$.

\subsubsection{Alcohol}

Studies investigating the influence of alcohol on PTH levels have yielded conflicting results. Some studies have found a decrease in PTH levels in alcoholics, while most studies have not reported a significant change in PTH levels due to alcohol consumption (Table 1). 
Moreover, the two largest studies involving more than 7000 participants yielded conflicting results; Jorde et al. observed a significant reduction in PTH levels in alcoholics [30], while Paik et al. did not notice a significant change in PTH levels in alcoholics [32]. Because alcohol inhibits bone regeneration [89], it has been suggested that alcohol intake reduces PTH levels [90-92] and increases calcitonin levels [93].

Several studies investigated calcitonin levels in alcoholics, and all yielded conflicting results (Table 2) with calcitonin levels that were increased [94], decreased [95], or unchanged [96] in alcoholics. Schuster et al. suggested that the reduction in calcitonin in chronic alcoholism is due to lower calcium concentration at this stage of alcohol consumption [95]. Interestingly, animal studies have shown that salmon calcitonin intake reduces various alcohol-related behaviors [97,98].

\subsubsection{Exercise}

Most studies that have investigated the influence of exercise on PTH levels have reported an increase in PTH levels during and after exercise (Table 1). However, most of these studies involved a small number of participants (less than 50). In contrast to the results of these studies, two studies involving as many as 7561 [31] and 3427 [30] participants reported a decrease in PTH levels after exercise. Causes of inconsistencies between studies may be the physical status of the participants; the age and gender of the participants; and the type, duration, and intensity of the exercise [99]. PTH is thought to increase during highintensity exercise (reviewed in [100]). Although exercise is thought to be beneficial for BMD, some groups of professional athletes have had significant reductions in BMD [101,102]. It has been suggested that intense exercise leads to a decrease in calcium levels, resulting in an increase in PTH. Elevated PTH levels may contribute to bone resorption (reviewed in [103]). Moreover, Shea et al. suggested that calcium supplementation during exercise could reduce bone resorption [104]. However, other researchers have noticed an increase in PTH levels during exercise despite the stability of calcium levels (reviewed in [103]). Some other factors that can lead to an increase in PTH during exercise are increased catecholamine release (which stimulates PTH release) [105], increased aldosterone release (which increases PTH and calcitonin release) [80], and acidosis (stimulates PTH release) [106].

Calcitonin levels increased $[107,108]$ or did not change [20,109-112] during exercise. However, these results should be verified in larger cohorts as most of these studies involved less than 30 participants (Table 2). Calcitonin levels could increase during exercise due to an increase in aldosterone levels [80]. 
Table 1. Lifestyle factors that affect PTH levels in humans.

\begin{tabular}{|c|c|c|c|c|c|}
\hline & Factor & $\begin{array}{l}\text { Effect on } \\
\text { Hormone Levels }\end{array}$ & Number of Participants & Participants & Reference \\
\hline \multirow{35}{*}{ की } & Smoking & $\downarrow \mathrm{PTH}$ & 170 (men) & Healthy adults & [113] \\
\hline & Smoking & $\downarrow \mathrm{PTH}$ & 376 & Healthy adults & [114] \\
\hline & Smoking & $\downarrow \mathrm{PTH}$ & 510 & Healthy adults & [62] \\
\hline & Smoking & $\leftrightarrow \mathrm{PTH}$ & 535 & Healthy adults & [115] \\
\hline & Smoking & $\leftrightarrow \mathrm{PTH}$ & 1203 & Healthy adults & [116] \\
\hline & Smoking & $\downarrow \mathrm{iPTH}$ & 177 & Healthy adults & [117] \\
\hline & Smoking & $\begin{array}{l}\downarrow P T H \text { (in mothers } \\
\text { and their } \\
\text { new-borns) }\end{array}$ & 61 & $\begin{array}{l}\text { Mothers and their } \\
\text { new-borns }\end{array}$ & {$[33]$} \\
\hline & Smoking & $\downarrow \mathrm{iPTH}$ & 31 (men) & Healthy adults & [118] \\
\hline & Smoking & $\leftrightarrow \mathrm{iPTH}$ & 43 (women) & Healthy adults & [118] \\
\hline & Smoking & $\downarrow \mathrm{PTH}$ & 7896 & Healthy adults & [30] \\
\hline & Smoking & $\downarrow \mathrm{PTH}$ & 405 (women) & Healthy adults & {$[37]$} \\
\hline & Smoking & $\downarrow \mathrm{PTH}$ & 958 (men) & Healthy adults & [119] \\
\hline & Smoking & $\leftrightarrow \mathrm{PTH}$ & 136 & Healthy adults & [92] \\
\hline & Smoking & $\downarrow \mathrm{PTH}$ & 406 & Healthy adults & {$[38]$} \\
\hline & Smoking & $\leftrightarrow \mathrm{PTH}$ & 3212 & $\begin{array}{l}2758 \text { healthy adults }+ \\
454 \text { participants with } \\
\text { coronary heart disease }\end{array}$ & [120] \\
\hline & Smoking & $\downarrow \mathrm{iPTH}$ & 347 & Healthy adults & [61] \\
\hline & Smoking & $\leftrightarrow \mathrm{PTH}$ & 1206 & Healthy adults & [121] \\
\hline & Smoking & $\leftrightarrow \mathrm{PTH}$ & 1068 & Healthy adults & [122] \\
\hline & Smoking & $\downarrow \mathrm{iPTH}$ & 345 & $\begin{array}{l}216 \text { healthy adults }+ \\
129 \text { men with earlier } \\
\text { partial gastrectomy }\end{array}$ & [123] \\
\hline & Smoking & $\downarrow \mathrm{PTH}$ & 7561 & Healthy adults & [31] \\
\hline & Smoking & $\downarrow$ iPTH & 3949 & Healthy adults & [124] \\
\hline & Smoking & $\leftrightarrow \mathrm{PTH}$ & 32 & Healthy adults & [125] \\
\hline & Smoking & $\downarrow \mathrm{PTH}$ & 1288 & Healthy adults & [63] \\
\hline & Smoking & $\downarrow \mathrm{PTH}$ & 7652 & Healthy adults & {$[32]$} \\
\hline & Smoking & $\leftrightarrow \mathrm{PTH}$ & 414 & Healthy adults & [126] \\
\hline & Smoking & $\downarrow \mathrm{PTH}$ & 2810 & Healthy adults & [127] \\
\hline & Smoking & $\leftrightarrow \mathrm{PTH}$ & 1205 & Healthy adults & [128] \\
\hline & & $\leftrightarrow \mathrm{PTH}$ & 719 (men) & & \\
\hline & Smoking & $\uparrow \mathrm{PTH}$ & $\begin{array}{l}128 \text { (participants } \\
\text { with low } \\
\text { body weight }(\leq 75 \mathrm{~kg}))\end{array}$ & Healthy adults & [129] \\
\hline & Smoking & $\downarrow \mathrm{PTH}$ & 1067 (women) & Healthy adults & [130] \\
\hline & Smoking & $\downarrow \mathrm{PTH}$ & 47 (women) & Healthy adults & [131] \\
\hline & Smoking & $\leftrightarrow \mathrm{PTH}$ & 489 (women) & Healthy adults & [91] \\
\hline & Smoking & $\downarrow \mathrm{PTH}$ & 908 & Healthy adults & [132] \\
\hline & Smoking & $\downarrow \mathrm{PTH}$ & 294 (women) & Healthy adults & [18] \\
\hline & Smoking & $\leftrightarrow \mathrm{PTH}$ & 58 & Healthy adults & [133] \\
\hline
\end{tabular}


Table 1. Cont.

\begin{tabular}{|c|c|c|c|c|c|}
\hline & Factor & $\begin{array}{l}\text { Effect on } \\
\text { Hormone Levels }\end{array}$ & Number of Participants & Participants & Reference \\
\hline \multirow{20}{*}{ ר: } & Alcohol & $\leftrightarrow \mathrm{PTH}$ & 535 & Healthy adults & [115] \\
\hline & Alcohol & $\leftrightarrow \mathrm{PTH}$ & 510 & Healthy adults & {$[62]$} \\
\hline & Alcohol & $\leftrightarrow \mathrm{PTH}$ & 1203 & Healthy adults & [116] \\
\hline & Alcohol & $\downarrow \mathrm{PTH}$ & 7896 & Healthy adults & {$[30]$} \\
\hline & Alcohol & $\downarrow \mathrm{PTH}$ & 136 & Healthy adults & {$[92]$} \\
\hline & Alcohol & $\leftrightarrow \mathrm{PTH}$ & 1206 & Healthy adults & [121] \\
\hline & Alcohol & $\downarrow$ iPTH & 3949 & Healthy adults & [124] \\
\hline & Alcohol & $\leftrightarrow \mathrm{PTH}$ & 1288 & Healthy adults & [63] \\
\hline & Alcohol & $\leftrightarrow \mathrm{PTH}$ & 414 & Healthy adults & [126] \\
\hline & Alcohol & $\leftrightarrow \mathrm{PTH}$ & 1205 & Healthy adults & [128] \\
\hline & Alcohol & $\leftrightarrow \mathrm{PTH}$ & 7652 & Healthy adults & [32] \\
\hline & Alcohol & $\leftrightarrow \mathrm{PTH}$ & 27 (men) & Healthy adults, alcoholics & [134] \\
\hline & Alcohol & $\leftrightarrow \mathrm{PTH}$ & 21 (men) & Healthy adults, alcoholics & {$[135]$} \\
\hline & Alcohol & $\downarrow \mathrm{PTH}$ & 6 & Healthy adults & [90] \\
\hline & Alcohol & $\leftrightarrow \mathrm{PTH}$ & 47 & Healthy adults, alcoholics & [95] \\
\hline & Alcohol & $\leftrightarrow \mathrm{PTH}$ & 26 & Healthy adults & [136] \\
\hline & Alcohol & $\downarrow \mathrm{PTH}$ & 136 & Healthy adults & [92] \\
\hline & Alcohol & $\begin{array}{l}\downarrow \text { PTH (increase in } \\
\text { PTH levels after al- } \\
\text { cohol withdrawal) }\end{array}$ & 26 & Healthy adults, alcoholics & [137] \\
\hline & Alcohol & $\leftrightarrow \mathrm{iPTH}$ & 36 (men) & Healthy adults, alcoholics & [138] \\
\hline & Alcohol & $\begin{array}{l}\downarrow \text { immunoreactive } \\
\text { PTH }\end{array}$ & 104 (men) & Healthy adults & [139] \\
\hline \multirow{29}{*}{ 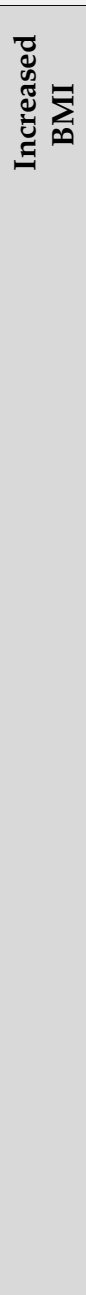 } & $\uparrow \mathrm{BMI}$ & $\leftrightarrow \mathrm{PTH}$ & 535 & Healthy adults & [115] \\
\hline & $\uparrow \mathrm{BMI}$ & $\uparrow \mathrm{PTH}$ & 510 & Healthy adults & [62] \\
\hline & $\uparrow \mathrm{BMI}$ & $\uparrow \mathrm{PTH}$ & 1203 & Healthy adults & {$[116]$} \\
\hline & $\uparrow \mathrm{BMI}$ & $\uparrow \mathrm{PTH}$ & 7896 & Healthy adults & [30] \\
\hline & $\uparrow \mathrm{BMI}$ & $\uparrow \mathrm{PTH}$ & 7561 & Healthy adults & [31] \\
\hline & $\uparrow \mathrm{BMI}$ & $\uparrow \mathrm{PTH}$ & 3212 & $\begin{array}{l}2758 \text { healthy adults }+ \\
454 \text { participants with } \\
\text { coronary heart disease }\end{array}$ & [120] \\
\hline & $\uparrow \mathrm{BMI}$ & $\uparrow \mathrm{iPTH}$ & 347 & Healthy adults & [61] \\
\hline & $\uparrow \mathrm{BMI}$ & $\uparrow \mathrm{PTH}$ & 1206 & Healthy adults & [121] \\
\hline & $\uparrow \mathrm{BMI}$ & $\uparrow \mathrm{PTH}$ & 2810 & Healthy adults & [127] \\
\hline & $\uparrow \mathrm{BMI}$ & $\uparrow \mathrm{PTH}$ & 1205 & Healthy adults & [128] \\
\hline & $\uparrow \mathrm{BMI}$ & $\uparrow \mathrm{PTH}$ & 7652 & Healthy adults & [32] \\
\hline & $\uparrow \mathrm{BMI}$ & $\uparrow \mathrm{PTH}$ & 1288 & Healthy adults & [63] \\
\hline & $\uparrow \mathrm{BMI}$ & $\uparrow \mathrm{iPTH}$ & 3949 & Healthy adults & [124] \\
\hline & $\uparrow \mathrm{BMI}$ & $\uparrow \mathrm{iPTH}$ & 160 & Healthy adults & [140] \\
\hline & $\uparrow \mathrm{BMI}$ & $\uparrow \mathrm{PTH}$ & 483 & Healthy adults & [141] \\
\hline & $\uparrow \mathrm{BMI}$ & $\leftrightarrow \mathrm{PTH}$ & 57 & Healthy adults & [79] \\
\hline & $\uparrow \mathrm{BMI}$ & $\uparrow \mathrm{PTH}$ & 57 (men) & Healthy adults & [142] \\
\hline & $\uparrow \mathrm{BMI}$ & $\uparrow \mathrm{PTH}$ & 1628 & Dialysis patients & [143] \\
\hline & $\uparrow \mathrm{BMI}$ & $\uparrow \mathrm{PTH}$ & 419 & Children & {$[144]$} \\
\hline & $\uparrow \mathrm{BMI}$ & $\uparrow \mathrm{PTH}$ & 82 (women) & Healthy adults & [145] \\
\hline & $\uparrow \mathrm{BMI}$ & $\uparrow \mathrm{PTH}$ & 316 & Healthy adults & [146] \\
\hline & $\uparrow \mathrm{BMI}$ & $\uparrow \mathrm{iPTH}$ & 332 & Healthy adults & [147] \\
\hline & $\uparrow \mathrm{BMI}$ & $\uparrow \mathrm{PTH}$ & 40 & $\begin{array}{l}\text { Bariatric surgery patients } \\
\text { and healthy controls }\end{array}$ & [148] \\
\hline & $\uparrow \mathrm{BMI}$ & $\uparrow \mathrm{PTH}$ & 316 & $\begin{array}{l}\text { Patients who had attended } \\
\text { the obesity clinics }\end{array}$ & [149] \\
\hline & $\uparrow \mathrm{BMI}$ & $\uparrow \mathrm{PTH}$ & 42 & $\begin{array}{l}\text { Patients undergoing } \\
\text { sleeve gastrectomy }\end{array}$ & [150] \\
\hline & $\uparrow \mathrm{BMI}$ & $\uparrow \mathrm{PTH}$ & 516 & Healthy adults & [151] \\
\hline & $\uparrow \mathrm{BMI}$ & $\uparrow \mathrm{PTH}$ & 3248 (women) & Healthy adults & [152] \\
\hline & $\uparrow \mathrm{BMI}$ & $\uparrow \mathrm{PTH}$ & 669 (men) & Healthy adults & [153] \\
\hline & $\uparrow \mathrm{BMI}$ & $\uparrow \mathrm{iPTH}$ & 590 & Hemodialysis patients & [154] \\
\hline
\end{tabular}


Table 1. Cont.

\begin{tabular}{|c|c|c|c|c|c|}
\hline & Factor & $\begin{array}{l}\text { Effect on } \\
\text { Hormone Levels }\end{array}$ & Number of Participants & Participants & Reference \\
\hline & $\uparrow \mathrm{BMI}$ & $\uparrow \mathrm{PTH}$ & $\begin{array}{l}2758 \text { healthy adults }+ \\
454 \text { participants with } \\
\text { coronary heart disease }\end{array}$ & Healthy adults & [155] \\
\hline & $\uparrow \mathrm{BMI}$ & $\uparrow \mathrm{PTH}$ & 250 & Healthy adults & [156] \\
\hline & $\uparrow \mathrm{BMI}$ & $\uparrow \mathrm{PTH}$ & 608 & Healthy adults & {$[157]$} \\
\hline & $\uparrow \mathrm{BMI}$ & $\uparrow \mathrm{PTH}$ & 496 (men) & $\begin{array}{l}\text { Patients with chronic kidney } \\
\text { disease }\end{array}$ & [158] \\
\hline & $\uparrow \mathrm{BMI}$ & $\leftrightarrow \mathrm{PTH}$ & 1436 & Healthy adults & [159] \\
\hline & $\uparrow \mathrm{BMI}$ & $\uparrow \mathrm{PTH}$ & 304 (women) & Healthy adults & [160] \\
\hline & $\uparrow \mathrm{BMI}$ & $\uparrow \mathrm{PTH}$ & 156 & Obese children & {$[161]$} \\
\hline & $\uparrow \mathrm{BMI}$ & $\uparrow \mathrm{PTH}$ & 3002 & Healthy adults & [162] \\
\hline & $\uparrow \mathrm{BMI}$ & $\uparrow \mathrm{PTH}$ & 810 (women) & Healthy adults & [163] \\
\hline & & $\begin{array}{l}\uparrow \mathrm{PTH}(\mathrm{PTH}= \\
21.4-65.8 \mathrm{pg} / \mathrm{mL})\end{array}$ & 131 & $\begin{array}{l}\text { Healthy adults and subjects } \\
\text { with primary }\end{array}$ & \\
\hline & $\uparrow \mathrm{BMI}$ & $\begin{array}{l}\downarrow \mathrm{PTH}(\mathrm{PTH}= \\
147-2511.7 \mathrm{pg} / \mathrm{mL})\end{array}$ & 132 & hyperparathyroidism & {$[41]$} \\
\hline & $\uparrow \mathrm{BMI}$ & $\uparrow \mathrm{PTH}$ & 383 (women) & Healthy adults & [164] \\
\hline & $\uparrow \mathrm{BMI}$ & $\uparrow \mathrm{PTH}$ & 2848 & Healthy adults & [165] \\
\hline & $\uparrow \mathrm{BMI}$ & $\uparrow \mathrm{PTH}$ & 453 & Healthy adults & {$[166]$} \\
\hline & $\uparrow \mathrm{BMI}$ & $\uparrow \mathrm{PTH}$ & 25 & Anorexia nervosa patients & [167] \\
\hline & $\uparrow \mathrm{BMI}$ & $\uparrow \mathrm{PTH}$ & 98 & Healthy adults & [168] \\
\hline & $\uparrow \mathrm{BMI}$ & $\uparrow \mathrm{PTH}$ & 625 & Healthy adults & [71] \\
\hline & $\uparrow \mathrm{BMI}$ & $\uparrow \mathrm{PTH}$ & 294 & Healthy adults & [18] \\
\hline 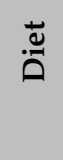 & $\begin{array}{l}\text { Different sorts of } \\
\text { vegetables, sausages, } \\
\text { salami, mushrooms, } \\
\text { eggs, white bread }\end{array}$ & $\uparrow \mathrm{PTH}$ & 1180 & Healthy adults & {$[21]$} \\
\hline & Bran bread & $\downarrow \mathrm{PTH}$ & & & \\
\hline & $\begin{array}{l}\text { Traditional Inuit diet } \\
\text { (diet mainly of marine } \\
\text { origin taken by } \\
\text { Greenland inhabitants) }\end{array}$ & $\downarrow \mathrm{PTH}$ & 535 & Healthy adults & [115] \\
\hline & $\uparrow$ Total calorie intake & $\leftrightarrow \mathrm{iPTH}$ & 3949 & Healthy adults & [124] \\
\hline & Protein intake & $\leftrightarrow \mathrm{PTH}$ & 7652 & Healthy adults & {$[32]$} \\
\hline & $\begin{array}{l}\text { Coronary Health } \\
\text { Improvement Project } \\
\text { (CHIP). CHIP } \\
\text { intervention, which } \\
\text { promotes a plant-based } \\
\text { diet with little dairy } \\
\text { intake and meat } \\
\text { consumption }\end{array}$ & $\begin{array}{l}\uparrow \mathrm{PTH} \text { (after } \\
6 \text { weeks) }\end{array}$ & 119 (women) & Healthy adults & [57] \\
\hline & $\begin{array}{l}\text { High-phosphorus, } \\
\text { low-calcium diets }\end{array}$ & $\uparrow \mathrm{PTH}$ & 16 & Healthy adults & {$[50]$} \\
\hline & $\begin{array}{l}\text { The traditional Brazilian } \\
\text { diet (fruits, vegetables, } \\
\text { and small amounts } \\
\text { of meat) }\end{array}$ & $\downarrow \mathrm{PTH}$ & 111 & Severely obese adults & [169] \\
\hline & $\begin{array}{l}\text { Extra virgin olive oil } \\
\text { supplementation }\end{array}$ & $\leftrightarrow \mathrm{PTH}$ & 111 & Severely obese adults & [169] \\
\hline & $\begin{array}{l}\text { Moderate dietary } \\
\text { protein restriction }\end{array}$ & $\uparrow \mathrm{PTH}$ & 18 & $\begin{array}{l}\text { Patients with idiopathic } \\
\text { hypercalciuria and } \\
\text { calcium nephrolithiasis }\end{array}$ & [55] \\
\hline & Vegans vs omnivores & $\uparrow \mathrm{PTH}$ in vegans & 155 & Healthy adults & {$[58]$} \\
\hline
\end{tabular}


Table 1. Cont.

\begin{tabular}{|c|c|c|c|c|}
\hline Factor & $\begin{array}{l}\text { Effect on } \\
\text { Hormone Levels }\end{array}$ & Number of Participants & Participants & Reference \\
\hline $\begin{array}{l}\text { The "Dietary } \\
\text { Approaches to Stop } \\
\text { Hypertension" (DASH) } \\
\text { diet, rich in fiber and } \\
\text { low-fat dairy }\end{array}$ & $\leftrightarrow \mathrm{PTH}$ & 334 & Healthy adults & [170] \\
\hline Vegans vs. omnivores & $\leftrightarrow \mathrm{PTH}$ & 210 (women) & Healthy adults & {$[171]$} \\
\hline $\begin{array}{l}\text { High protein and high } \\
\text { dairy group }\end{array}$ & $\downarrow \mathrm{PTH}$ & 30 (women) & Healthy adults & {$[56]$} \\
\hline $\begin{array}{l}\text { Adequate protein and } \\
\text { medium dairy group }\end{array}$ & $\downarrow \mathrm{PTH}$ & 30 (women) & Healthy adults & [56] \\
\hline $\begin{array}{l}\text { Adequate protein and } \\
\text { low dairy }\end{array}$ & $\uparrow \mathrm{PTH}$ & 30 (women) & Healthy adults & {$[56]$} \\
\hline $\begin{array}{l}\text { Diet with low } \\
\text { calcium:phosphorus ratio }\end{array}$ & $\uparrow \mathrm{PTH}$ & 147 (women) & Healthy adults & {$[51]$} \\
\hline $\begin{array}{l}\text { Low-protein diets (diets } \\
\text { containing } 0.7 \text { and } 0.8 \mathrm{~g} \\
\text { protein } / \mathrm{kg} \text { ) }\end{array}$ & $\uparrow \mathrm{PTH}$ & 8 (women) & Healthy adults & {$[54]$} \\
\hline $\begin{array}{l}\text { Higher consumption of } \\
\text { a proinflammatory diet }\end{array}$ & $\uparrow \mathrm{PTH}$ & 7679 & $\begin{array}{l}\text { Adults with/without } \\
\text { chronic kidney disease }\end{array}$ & {$[53]$} \\
\hline $\begin{array}{l}\text { High fruit and vegetable } \\
\text { intake (consuming more } \\
\text { than } 3 \text { servings of fruit } \\
\text { and vegetables) }\end{array}$ & $\downarrow \mathrm{PTH}$ & 56 & Children & [172] \\
\hline $\begin{array}{l}\text { Dietary calorie, } \\
\text { vitamin } D \text {, and } \\
\text { magnesium intake }\end{array}$ & $\leftrightarrow \mathrm{PTH}$ & 98 & Healthy adults & [168] \\
\hline Vegetarians vs. controls & $\uparrow \mathrm{iPTH}$ & \multirow{3}{*}{44} & \multirow{3}{*}{ Healthy adults } & \multirow{3}{*}{ [59] } \\
\hline Intake of dietary fiber & $\uparrow \mathrm{iPTH}$ & & & \\
\hline Dietary calcium intake & $\downarrow$ iPTH & & & \\
\hline Coffee & $\downarrow$ iPTH & 181 (men) & Healthy adults & [61] \\
\hline Coffee, tea & $\leftrightarrow \mathrm{PTH}$ & 510 & Healthy adults & [62] \\
\hline Coffee & $\downarrow \mathrm{PTH}$ & 3427 (men) & Healthy adults & {$[30]$} \\
\hline Caffeine intake & $\leftrightarrow \mathrm{PTH}$ & 7652 & Healthy adults & [32] \\
\hline Caffeine intake & $\leftrightarrow \mathrm{PTH}$ & 1288 & Healthy adults & [63] \\
\hline Vitamin D supplements & $\leftrightarrow \mathrm{PTH}$ & 510 & Healthy adults & [62] \\
\hline Vitamin D supplements & $\downarrow \mathrm{PTH}$ & 4469 (women) & Healthy adults & [30] \\
\hline Vitamin D supplements & $\downarrow$ iPTH & 3949 & Healthy adults & {$[124]$} \\
\hline Vitamin D supplements & $\leftrightarrow \mathrm{PTH}$ & 1288 & Healthy adults & [63] \\
\hline Vitamin D supplements & $\downarrow \mathrm{PTH}$ & 414 & Healthy adults & {$[126]$} \\
\hline Vitamin D intake & $\downarrow \mathrm{PTH}$ & 316 & Healthy adults & [146] \\
\hline $\begin{array}{l}\text { Vitamin D } \\
\text { supplementation }\end{array}$ & $\downarrow \mathrm{PTH}$ & 250 & Healthy adults & [156] \\
\hline Vitamin D intake & $\downarrow$ PTH & 376 (women) & Healthy adults & {$[173]$} \\
\hline $\begin{array}{l}\text { Vitamin D } \\
\text { supplementation }\end{array}$ & $\downarrow \mathrm{PTH}$ & Meta-analysis & & {$[70]$} \\
\hline $\begin{array}{l}\text { Vitamin D and calcium } \\
\text { supplementation }\end{array}$ & $\downarrow \mathrm{PTH}$ & 77 & Healthy adults & [174] \\
\hline $\begin{array}{l}\text { Vitamin D and calcium } \\
\text { supplementation }\end{array}$ & $\downarrow \mathrm{PTH}$ & 247 (women) & Healthy adults & [175] \\
\hline $\begin{array}{l}\text { Vitamin D and calcium } \\
\text { supplementation }\end{array}$ & $\downarrow \mathrm{PTH}$ & 877 (women) & Healthy adults & [176] \\
\hline $\begin{array}{l}\text { Vitamin D } \\
\text { supplementation }\end{array}$ & $\downarrow \mathrm{PTH}$ & 270 (women) & Healthy adults & {$[75]$} \\
\hline $\begin{array}{l}\text { Vitamin D and calcium } \\
\text { supplementation }\end{array}$ & $\downarrow \mathrm{PTH}$ & 313 & Healthy adults & [177] \\
\hline $\begin{array}{l}\text { Vitamin D and calcium } \\
\text { supplementation }\end{array}$ & $\downarrow \mathrm{PTH}$ & 103 (women) & $\begin{array}{l}\text { Elderly institutionalised } \\
\text { women }\end{array}$ & [178] \\
\hline
\end{tabular}


Table 1. Cont.

\begin{tabular}{|c|c|c|c|c|}
\hline Factor & $\begin{array}{l}\text { Effect on } \\
\text { Hormone Levels }\end{array}$ & Number of Participants & Participants & Reference \\
\hline $\begin{array}{l}\text { Vitamin D } \\
\text { supplementation }\end{array}$ & $\leftrightarrow \mathrm{PTH}$ & 128 (women) & Healthy adults & [179] \\
\hline $\begin{array}{l}\text { Vitamin D and calcium } \\
\text { supplementation }\end{array}$ & $\downarrow \mathrm{PTH}$ & 145 (women) & Healthy adults & [180] \\
\hline $\begin{array}{l}\text { Vitamin D } \\
\text { supplementation }\end{array}$ & $\downarrow \mathrm{PTH}$ & 60 (men) & Healthy adults & {$[181]$} \\
\hline $\begin{array}{l}\text { Vitamin D and calcium } \\
\text { supplementation }\end{array}$ & $\downarrow \mathrm{PTH}$ & 192 (women) & Healthy adults & [182] \\
\hline $\begin{array}{l}\text { Vitamin D and calcium } \\
\text { supplementation }\end{array}$ & $\downarrow \mathrm{PTH}$ & 191 (women) & Ambulatory elderly women & [183] \\
\hline $\begin{array}{l}\text { Vitamin D } \\
\text { supplementation }\end{array}$ & $\leftrightarrow \mathrm{PTH}$ & 208 (women) & Healthy adults & [184] \\
\hline $\begin{array}{l}\text { Vitamin D and calcium } \\
\text { supplementation }\end{array}$ & $\downarrow \mathrm{PTH}$ & 314 & Healthy adults & [185] \\
\hline $\begin{array}{l}\text { Vitamin D and calcium } \\
\text { supplementation }\end{array}$ & $\downarrow \mathrm{PTH}$ & 1368 & Healthy adults & [127] \\
\hline $\begin{array}{l}\text { Vitamin D } \\
\text { supplementation }\end{array}$ & $\downarrow \mathrm{PTH}$ & 338 & Healthy adults & [186] \\
\hline $\begin{array}{l}\text { Vitamin D and calcium } \\
\text { supplementation }\end{array}$ & $\downarrow \mathrm{PTH}$ & 218 & Older patients & [187] \\
\hline $\begin{array}{l}\text { Vitamin D } \\
\text { supplementation }\end{array}$ & $\leftrightarrow \mathrm{PTH}$ & 215 & Healthy adults & [188] \\
\hline $\begin{array}{l}\text { Vitamin D and calcium } \\
\text { supplementation }\end{array}$ & $\downarrow \mathrm{PTH}$ & 242 & Healthy adults & [189] \\
\hline $\begin{array}{l}\text { Vitamin D } \\
\text { supplementation }\end{array}$ & $\downarrow \mathrm{PTH}$ & 165 & Healthy overweight subjects & [190] \\
\hline $\begin{array}{l}\text { Vitamin D and calcium } \\
\text { supplementation }\end{array}$ & $\downarrow \mathrm{PTH}$ & 153 & Healthy adults & [191] \\
\hline $\begin{array}{l}\text { Multiple micronutrient } \\
\text { and calcium } \\
\text { supplementation }\end{array}$ & $\downarrow \mathrm{PTH}$ & 153 (women) & Healthy adults & [191] \\
\hline $\begin{array}{l}\text { Vitamin D and calcium } \\
\text { supplementation }\end{array}$ & $\downarrow \mathrm{PTH}$ & 158 & Overweight subjects & [192] \\
\hline $\begin{array}{l}\text { Vitamin D } \\
\text { supplementation }\end{array}$ & $\downarrow \mathrm{PTH}$ & 202 & Healthy adults & [193] \\
\hline $\begin{array}{l}\text { Vitamin D } \\
\text { supplementation }\end{array}$ & $\downarrow \mathrm{PTH}$ & 94 & Healthy adults & [194] \\
\hline $\begin{array}{l}\text { Vitamin D } \\
\text { supplementation }\end{array}$ & $\leftrightarrow \mathrm{PTH}$ & 90 & $\begin{array}{l}\text { Coronary artery } \\
\text { disease patients }\end{array}$ & [195] \\
\hline $\begin{array}{l}\text { Vitamin D } \\
\text { supplementation }\end{array}$ & $\leftrightarrow \mathrm{PTH}$ & 151 & Healthy adults & [196] \\
\hline $\begin{array}{l}\text { Vitamin D } \\
\text { supplementation }\end{array}$ & $\downarrow \mathrm{PTH}$ & 89 & $\begin{array}{l}\text { Obese with pre- or } \\
\text { early diabetes }\end{array}$ & [197] \\
\hline $\begin{array}{l}\text { Vitamin D } \\
\text { supplementation }\end{array}$ & $\downarrow \mathrm{PTH}$ & 112 & Hypertensive patients & [198] \\
\hline $\begin{array}{l}\text { Vitamin D } \\
\text { supplementation }\end{array}$ & $\downarrow \mathrm{PTH}$ & 230 & Adults with depression & [199] \\
\hline $\begin{array}{l}\text { Vitamin D } \\
\text { supplementation }\end{array}$ & $\downarrow \mathrm{PTH}$ & 77 (women) & Healthy adults & [200] \\
\hline $\begin{array}{l}\text { Vitamin D and calcium } \\
\text { supplementation }\end{array}$ & $\downarrow \mathrm{PTH}$ & 173 (women) & Healthy adults & [201] \\
\hline $\begin{array}{l}\text { Vitamin D } \\
\text { supplementation }\end{array}$ & $\downarrow \mathrm{PTH}$ & 112 & Parkinson disease & [202] \\
\hline $\begin{array}{l}\text { Vitamin D } \\
\text { supplementation }\end{array}$ & $\leftrightarrow \mathrm{PTH}$ & 82 & Healthy adults & [203] \\
\hline Vitamin A intake & $\leftrightarrow \mathrm{PTH}$ & 606 & Healthy adults & {$[72]$} \\
\hline $\begin{array}{l}\text { Total calcium and } \\
\text { vitamin A intake }\end{array}$ & $\downarrow \mathrm{PTH}$ & 625 & Healthy adults & {$[71]$} \\
\hline
\end{tabular}


Table 1. Cont.

\begin{tabular}{|c|c|c|c|c|c|}
\hline & Factor & $\begin{array}{l}\text { Effect on } \\
\text { Hormone Levels }\end{array}$ & Number of Participants & Participants & Reference \\
\hline & Vitamin A intake & $\downarrow \mathrm{PTH}$ & 1288 & Healthy adults & {$[63]$} \\
\hline & $\begin{array}{l}\text { The dietary intake of } \\
\text { minerals (calcium, } \\
\text { phosphate, and } \\
\text { magnesium) and } \\
\text { vitamin D }\end{array}$ & $\leftrightarrow \mathrm{PTH}$ & 127 & Healthy adults & [204] \\
\hline & Calcium supplements & $\downarrow \mathrm{PTH}$ & 414 & Healthy adults & [126] \\
\hline & Calcium supplements & $\downarrow \mathrm{PTH}$ & 51 & Toddlers & [205] \\
\hline & Calcium intake & $\downarrow \mathrm{PTH}$ & 7896 & Healthy adults & [30] \\
\hline & Dietary calcium intake & $\downarrow \mathrm{PTH}$ & 181 & $\begin{array}{l}\text { Healthy } \\
\text { adolescents }\end{array}$ & [206] \\
\hline & Calcium intake & $\downarrow \mathrm{PTH}$ & 1203 & Healthy adults & [116] \\
\hline & Calcium intake & $\downarrow \mathrm{PTH}$ & 3212 & $\begin{array}{l}2758 \text { healthy adults }+ \\
454 \text { participants with } \\
\text { coronary heart disease }\end{array}$ & [120] \\
\hline & Calcium intake & $\leftrightarrow \mathrm{PTH}$ & 1288 & Healthy adults & [63] \\
\hline & Calcium intake & $\downarrow$ iPTH & 3949 & Healthy adults & [124] \\
\hline & Dietary calcium intake & $\downarrow \mathrm{PTH}$ & 7652 & Healthy adults & [32] \\
\hline & Calcium intake & $\leftrightarrow \mathrm{PTH}$ & 57 & Healthy adults & [79] \\
\hline & $\begin{array}{l}\text { Animal/total calcium } \\
\text { intake }\end{array}$ & $\downarrow \mathrm{PTH}$ & 316 & Healthy adults & [146] \\
\hline & Dietary calcium & $\leftrightarrow \mathrm{PTH}$ & 155 (women) & Healthy adults & [207] \\
\hline & Calcium supplements & $\downarrow \mathrm{PTH}$ & 566 & Healthy adults & [208] \\
\hline & Intake of calcium & $\downarrow$ PTH & 82 & Healthy adults & [203] \\
\hline & $\begin{array}{l}\text { Calcium intake derived } \\
\text { from milk }\end{array}$ & $\downarrow \mathrm{PTH}$ & 245 (women) & Healthy adults & [173] \\
\hline & Magnesium intake & $\leftrightarrow \mathrm{PTH}$ & 57 & Healthy adults & [79] \\
\hline & Magnesium intake & $\leftrightarrow \mathrm{PTH}$ & 7652 & Healthy adults & [32] \\
\hline & \multirow{2}{*}{$\begin{array}{l}\text { Magnesium } \\
\text { supplementation }\end{array}$} & $\uparrow \mathrm{PTH}$ & $\begin{array}{l}10 \text { (patients with } \\
\text { hypoparathyroidism) }\end{array}$ & \multirow{2}{*}{ Patients with osteoporosis } & \multirow{2}{*}{ [78] } \\
\hline & & $\downarrow \mathrm{PTH}$ & $\begin{array}{l}10 \text { (patients with } \\
\text { vitamin D insufficiency) }\end{array}$ & & \\
\hline & $\begin{array}{l}\text { Magnesium } \\
\text { supplementation }\end{array}$ & 个iPTH & 23 & Children with diabetes & [77] \\
\hline & Zinc infusion & $\leftrightarrow \mathrm{PTH}$ & 38 & $\begin{array}{l}\text { Patients of short stature, } \\
\text { diabetes mellitus, and } \\
\text { controls }\end{array}$ & [83] \\
\hline & Phosphorus intake & $\leftrightarrow \mathrm{PTH}$ & 7652 & Healthy adults & [32] \\
\hline & $\begin{array}{l}\text { Intervention group } \\
\text { (exercise, vitamin D, } \\
\text { calcium, protein } \\
\text { supplementation) }\end{array}$ & $\downarrow \mathrm{iPTH}$ & 220 & $\begin{array}{l}\text { Patients that were on } \\
\text { bariatric surgery }\end{array}$ & [209] \\
\hline \multirow{15}{*}{ 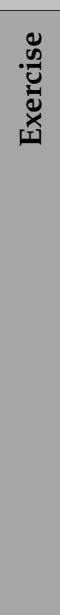 } & Exercise & $\downarrow \mathrm{PTH}$ & 7561 & Healthy adults & {$[31]$} \\
\hline & Exercise & $\leftrightarrow \mathrm{PTH}$ & 1288 & Healthy adults & {$[63]$} \\
\hline & Exercise & $\downarrow \mathrm{PTH}$ & 3427 (men) & Healthy adults & [30] \\
\hline & Exercise & $\leftrightarrow \mathrm{PTH}$ & 414 & Healthy adults & [126] \\
\hline & Exercise & $\leftrightarrow \mathrm{PTH}$ & 1205 & Healthy adults & [128] \\
\hline & $\uparrow$ Sitting & $\uparrow \mathrm{PTH}$ & 566 & Healthy adults & [208] \\
\hline & Exercise & $\downarrow \mathrm{PTH}$ & 625 & Healthy adults & {$[71]$} \\
\hline & Exercise & $\uparrow \mathrm{PTH}$ & 12 (men) & Healthy adults & [210] \\
\hline & Exercise & $\uparrow \mathrm{PTH}$ & 20 & Healthy adults & [211] \\
\hline & Exercise & $\downarrow \mathrm{PTH}$ & 54 & $\begin{array}{l}\text { Chronic kidney } \\
\text { disease patients }\end{array}$ & [212] \\
\hline & Exercise & $\uparrow \mathrm{PTH}$ & 29 & Boys and young men & [213] \\
\hline & Exercise & $\uparrow \mathrm{PTH}$ & 11 (men) & Healthy adults & {$[214]$} \\
\hline & Exercise & $\uparrow \mathrm{PTH}$ & 25 & Healthy adults & [215] \\
\hline & Exercise & $\uparrow \mathrm{PTH}$ & 12 (men) & Healthy adults & [216] \\
\hline & Exercise & $\leftrightarrow \mathrm{iPTH}$ & 100 (women) & Healthy adults & [217] \\
\hline
\end{tabular}


Table 1. Cont.

\begin{tabular}{|c|c|c|c|c|}
\hline Factor & $\begin{array}{l}\text { Effect on } \\
\text { Hormone Levels }\end{array}$ & Number of Participants & Participants & Reference \\
\hline Exercise & $\uparrow \mathrm{iPTH}$ & 21 & Healthy adults & [218] \\
\hline Exercise & $\uparrow \mathrm{iPTH}$ & 7 (men) & Healthy adults & [219] \\
\hline Exercise & $\downarrow \mathrm{PTH}$ & 5 (women) & Healthy adults & [220] \\
\hline Exercise & $\uparrow \mathrm{iPTH}$ & 9 (men) & Healthy adults & [221] \\
\hline Exercise & $\begin{array}{l}\uparrow \mathrm{PTH} \text { (during the } \\
\text { exercise with the } \\
\text { highest intensity) }\end{array}$ & 10 (men) & Healthy adults & [222] \\
\hline Exercise & $\begin{array}{l}\uparrow \mathrm{PTH} \text { (during } \\
\text { the exercise) } \\
\leftrightarrow \text { PTH } \\
\text { (postexercise } \\
\text { period) }\end{array}$ & 10 (men) & Healthy adults & [223] \\
\hline Exercise & $\uparrow \mathrm{PTH}$ & 10 (women) & Healthy adults & [104] \\
\hline Exercise & $\uparrow \mathrm{PTH}$ & 51 (men) & Healthy adults & {$[224]$} \\
\hline Exercise & $\begin{array}{l}\downarrow \text { iPTH (moderate } \\
\text { exercise) } \\
\uparrow i \text { TH (intensive } \\
\text { exercise) }\end{array}$ & 21 (women) & Healthy adults & [225] \\
\hline Exercise & $\uparrow \mathrm{PTH}$ & 14 (women) & Healthy adults & [226] \\
\hline Exercise & $\begin{array}{l}\downarrow \mathrm{PTH} \text { (with the } \\
\text { onset of exercise) } \\
\uparrow \mathrm{PTH} \text { (intensive } \\
\text { exercise) }\end{array}$ & 10 (men) & Healthy adults & [227] \\
\hline Exercise & $\uparrow \mathrm{PTH}$ & 17 (men) & Healthy adults & [228] \\
\hline Exercise & $\uparrow \mathrm{PTH}$ & 100 (men) & Healthy adults & [229] \\
\hline Exercise & $\uparrow \mathrm{PTH}$ & 9 (men) & Healthy adults & [111] \\
\hline Exercise & $\uparrow \mathrm{PTH}$ & 26 (women) & Healthy adults & [230] \\
\hline Exercise & $\uparrow \mathrm{PTH}$ & 18 & Healthy adults & [112] \\
\hline Exercise & $\uparrow \mathrm{iPTH}$ & 8 (men) & Healthy adults & [231] \\
\hline Exercise & $\leftrightarrow \mathrm{PTH}$ & 6 (men) & Healthy adults & [232] \\
\hline Exercise & $\uparrow \mathrm{PTH}$ & 6 (men) & Healthy adults & [109] \\
\hline Exercise & $\uparrow \mathrm{PTH}$ & 19 (men) & Healthy adults & [107] \\
\hline Exercise & $\leftrightarrow \mathrm{PTH}$ & 13 (men) & Healthy adults & [110] \\
\hline Exercise & $\uparrow$ PTH & 27 (men) & Healthy adults & [20] \\
\hline
\end{tabular}

BMI, body mass index; iPTH, intact parathyroid hormone; PTH, parathyroid hormone. Decreased $(\downarrow)$, unchanged $(\leftrightarrow)$, increased $(\uparrow)$.

Table 2. Lifestyle factors that affect calcitonin levels in humans.

\begin{tabular}{|c|c|c|c|c|c|}
\hline & Factor & $\begin{array}{l}\text { Effect on } \\
\text { Hormone Levels }\end{array}$ & $\begin{array}{l}\text { Number of } \\
\text { Participants }\end{array}$ & Participants & Reference \\
\hline \multirow{6}{*}{ की } & Smoking & $\leftrightarrow$ Calcitonin & 294 (women) & Healthy adults & [18] \\
\hline & Smoking & $\uparrow$ Calcitonin & 9340 & People with type 2 diabetes & {$[48]$} \\
\hline & Smoking & $\uparrow$ Calcitonin & 142 (men) & Healthy adults & [233] \\
\hline & Smoking & $\uparrow$ Calcitonin & 58 & Healthy adults & [133] \\
\hline & Smoking & $\uparrow$ Calcitonin & 120 (men) & Healthy adults & [234] \\
\hline & Smoking & $\uparrow$ Calcitonin & 6341 (men) & Healthy adults & [17] \\
\hline \multirow{4}{*}{ 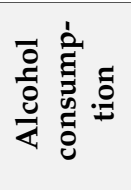 } & Alcohol & $\leftrightarrow$ Calcitonin & 26 & Healthy adults & [136] \\
\hline & Alcohol & $\leftrightarrow$ Calcitonin & 93 & Healthy adults & [96] \\
\hline & Alcohol & $\begin{array}{l}\downarrow \text { Calcitonin (in a heavy } \\
\text { drinking group) }\end{array}$ & 47 & Alcoholics & [95] \\
\hline & Alcohol & $\uparrow$ Calcitonin & 50 & Alcoholics + controls & [94] \\
\hline
\end{tabular}


Table 2. Cont.

\begin{tabular}{|c|c|c|c|c|c|}
\hline & Factor & $\begin{array}{l}\text { Effect on } \\
\text { Hormone Levels }\end{array}$ & $\begin{array}{l}\text { Number of } \\
\text { Participants }\end{array}$ & Participants & Reference \\
\hline \multirow{6}{*}{ 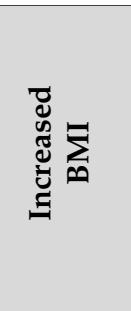 } & $\uparrow \mathrm{BMI}$ & $\leftrightarrow$ Calcitonin & 467 & $\begin{array}{l}\text { Patients with Hashimoto's } \\
\text { thyroiditis }\end{array}$ & [235] \\
\hline & $\uparrow \mathrm{BMI}$ & $\downarrow$ Calcitonin & 294 & Healthy adults & [18] \\
\hline & $\uparrow \mathrm{BMI}$ & $\uparrow$ Calcitonin & 9340 & People with type 2 diabetes & [48] \\
\hline & $\uparrow \mathrm{BMI}$ & $\uparrow$ Calcitonin & 287 & Healthy adults & [233] \\
\hline & $\uparrow \mathrm{BMI}$ & $\leftrightarrow$ Calcitonin & 4638 & Healthy adults & [17] \\
\hline & $\uparrow \mathrm{BMI}$ & $\uparrow$ Calcitonin & 31 & $\begin{array}{l}\text { Patients with chronic kidney } \\
\text { disease on hemodialysis }\end{array}$ & [236] \\
\hline \multirow{4}{*}{ 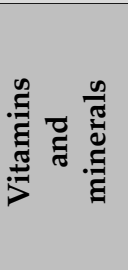 } & $\begin{array}{l}\text { Vitamin D } \\
\text { supplementation }\end{array}$ & $\leftrightarrow$ Calcitonin & 270 (women) & Healthy adults & [75] \\
\hline & Zinc infusion & $\downarrow$ Calcitonin & 38 & $\begin{array}{l}\text { Patients of short stature, } \\
\text { diabetes mellitus, } \\
\text { and controls }\end{array}$ & [83] \\
\hline & High dietary zinc & $\downarrow$ Calcitonin & 21 & Healthy adults & [86] \\
\hline & High dietary copper & $\leftrightarrow$ Calcitonin & 21 & Healthy adults & {$[86]$} \\
\hline \multirow{7}{*}{ 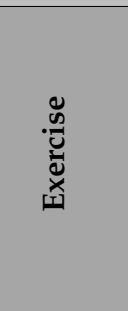 } & Exercise & $\leftrightarrow$ Calcitonin & 9 (men) & Healthy adults & [111] \\
\hline & Exercise & $\leftrightarrow$ Calcitonin & 18 & Healthy adults & [112] \\
\hline & Exercise & $\leftrightarrow$ Calcitonin & 6 (men) & Healthy adults & [109] \\
\hline & Exercise & $\uparrow$ Calcitonin & 19 (men) & Healthy adults & [107] \\
\hline & Exercise & $\leftrightarrow$ Calcitonin & 13 (men) & Healthy adults & [110] \\
\hline & Exercise & $\leftrightarrow$ Calcitonin & 27 (men) & Healthy adults & [20] \\
\hline & $\begin{array}{l}\text { Raloxifene combined } \\
\text { with aerobic exercise }\end{array}$ & $\uparrow$ Calcitonin & 70 & Patients with osteoporosis & [108] \\
\hline
\end{tabular}

BMI, body mass index. Decreased $(\downarrow)$, unchanged $(\leftrightarrow)$, increased $(\uparrow)$.

\subsection{Pollutants}

\subsubsection{Heavy Metals}

Various heavy metals, such as cadmium (Cd), arsenic (As), and lead (Pb), affect PTH levels. Most studies have shown that PTH levels decrease after cadmium exposure (Table 3). Schutte et al., explained the decrease in PTH levels after cadmium exposure as a consequence of the direct osteotoxic effect of cadmium [18]. Exposure to cadmium leads to a decrease in bone density, resulting in increased release of calcium from bone tissue. The result of increased calcium release is the decrease in PTH levels [18]. In addition, cadmium has been shown to have a toxic effect on parathyroid glands [237]. However, some studies did not observe any effect [238-240] or observed an increase [241,242] in PTH levels in subjects exposed to cadmium. Studies in experimental animals observed an increase in PTH levels after cadmium exposure [243]. Arsenic exposure did not affect PTH levels [244]. Most studies reported an increase in PTH levels in subjects exposed to lead (Table 3). Lead inhibits $1 \alpha$-hydroxylase (the enzyme responsible for the production of $1,25(\mathrm{OH}) 2 \mathrm{D})$ [245], and since PTH and $25(\mathrm{OH}) \mathrm{D}$ are in an inverse relationship, a decrease in $25(\mathrm{OH}) \mathrm{D}$ levels results in an increase in PTH levels. PTH levels were also measured in Gulf War I veterans who were exposed to uranium, and it was shown that uranium exposure led to a decrease in PTH levels [246].

We found only one study that analyzed the influence of heavy metals on calcitonin levels. Schutte et al., observed an increase in calcitonin levels after cadmium exposure [18]. A study in rats showed that exposure to cadmium and lead decreased calcitonin levels [243,247]. Exposure of laying hens to cadmium led to a decrease in calcitonin levels [248], while a study in goldfish found no changes in calcitonin levels after cadmium exposure (although exposure to methylmercury increased calcitonin levels) [249].

\subsubsection{Chemicals}

Only a few studies have investigated the effect of chemicals on PTH levels in humans (Table 3). Exposure to persistent organochlorine compounds ( $\mathrm{p}, \mathrm{p}^{\prime}$-diphenyldichloroethene (p, $\mathrm{p}^{\prime}$-DDE) and polychlorinated biphenyls (PCBs)) did not affect PTH levels [132,250]. 
Exposure to perfluoroalkyl substances (PFAS) led to an increase in PTH levels [251]. Di Nisio et al. suggested that perfluoro-octanoic acid (PFOA) binds to vitamin D receptors, causing reduced 1,25(OH)D activity, which in turn increases PTH levels [251]. Fluoride exposure increases PTH levels [252]. According to researchers, excess fluoride alters calcium metabolism and potentially leads to secondary hyperparathyroidism (reviewed in [253]). Exposure to perchlorate, thiocyanate, and nitrate has led to a decrease in PTH levels, but the underlying mechanism of this action is not yet clear [19].

Data on the effect of chemicals and pesticides on calcitonin levels in humans are scarce. A study on goldfish has shown that bisphenol A inhibits the release of calcitonin [249]. Aroclor 1254 (PCB) increased calcitonin expression in rat thyroid [254]. Because many chemicals have an endocrine disruptive effect [255], further studies are needed on the impact of chemicals and pesticides on PTH and calcitonin levels.

Table 3. Pollutants affecting PTH and calcitonin levels in humans.

\begin{tabular}{|c|c|c|c|c|c|}
\hline & Factor & $\begin{array}{c}\text { Effect on } \\
\text { Hormone Levels }\end{array}$ & $\begin{array}{l}\text { Number of } \\
\text { Participants }\end{array}$ & Participants & Reference \\
\hline \multirow{20}{*}{ 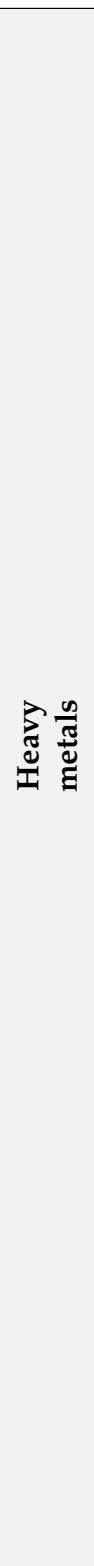 } & Arsenic & $\leftrightarrow$ PTH- & 196 & Healthy adults & [256] \\
\hline & Arsenic & $\leftrightarrow \mathrm{iPTH}$ & 774 & Children and new-borns & [244] \\
\hline & Cadmium & $\downarrow$ PTH & 719 (women) & Healthy adults & {$[34]$} \\
\hline & Cadmium & $\downarrow \mathrm{PTH}$ & 85 (women) & Healthy adults & [257] \\
\hline & Cadmium & $\downarrow \mathrm{PTH}$ & 51 (men) & $\begin{array}{l}\text { Participants exposed } \\
\text { to cadmium }\end{array}$ & [258] \\
\hline & Cadmium & $\leftrightarrow \mathrm{PTH}$ & 46 & $\begin{array}{l}\text { Participants exposed to } \\
\text { cadmium for a long period } \\
\text { (some suffering } \\
\text { from decreased } \\
\text { tubular function) }\end{array}$ & [240] \\
\hline & Cadmium & $\leftrightarrow \mathrm{PTH}$ & 41 (women) & $\begin{array}{l}\text { Subjects with renal tubular } \\
\text { dysfunction caused by } \\
\text { exposure to cadmium }\end{array}$ & [259] \\
\hline & Cadmium & $\downarrow \mathrm{iPTH}$ & 306 & $\begin{array}{l}\text { Chronic peritoneal dialysis } \\
\text { patients }\end{array}$ & [260] \\
\hline & $\begin{array}{l}\text { Cadmium in urine } \\
\text { (maternal) }\end{array}$ & $\begin{array}{l}\downarrow \mathrm{PTH} \text { (in boys) } \\
\uparrow \mathrm{PTH} \text { (in girls) }\end{array}$ & 504 & \multirow[b]{2}{*}{$\begin{array}{l}504 \text { children in a } \\
\text { mother-child cohort }\end{array}$} & \multirow[b]{2}{*}{ [242] } \\
\hline & $\begin{array}{l}\text { Cadmium in } \\
\text { erythrocytes } \\
\text { (maternal) }\end{array}$ & $\begin{array}{l}\uparrow \mathrm{PTH} \text { (in boys) } \\
\downarrow \text { PTH (in girls) }\end{array}$ & 504 & & \\
\hline & Cadmium & $\leftrightarrow \mathrm{PTH}$ & 60 & $\begin{array}{l}\text { Patients with renal tubular } \\
\text { damage caused by exposure } \\
\text { to cadmium and } \\
\text { healthy controls }\end{array}$ & [238] \\
\hline & Cadmium & $\uparrow \mathrm{PTH}$ & 53 & $\begin{array}{l}\text { Patients with renal tubular } \\
\text { damage caused by exposure } \\
\text { to cadmium and } \\
\text { healthy controls }\end{array}$ & [241] \\
\hline & Cadmium & $\begin{array}{c}\downarrow \mathrm{PTH} \text { (association lost } \\
\text { after adjustment } \\
\text { for smoking) }\end{array}$ & 908 (women) & Healthy adults & [132] \\
\hline & Cadmium & $\begin{array}{c}\downarrow \mathrm{PTH}, \\
\uparrow \text { Calcitonin }\end{array}$ & 294 (women) & Healthy adults & [18] \\
\hline & Cadmium & $\leftrightarrow$ PTH & 146 & Healthy adults & [239] \\
\hline & Lead & $\uparrow \mathrm{PTH}$ & 89 & Healthy adults & [245] \\
\hline & Lead & $\leftrightarrow \mathrm{PTH}$ & 719 (women) & Healthy adults & [34] \\
\hline & Lead & $\leftrightarrow \mathrm{PTH}$ & 51 & Dialysis patients & [261] \\
\hline & Lead & $\uparrow \mathrm{PTH}$ & 146 (men) & Healthy adults & [262] \\
\hline & Lead & $\uparrow \mathrm{iPTH}$ & 315 & $\begin{array}{l}\text { Chronic peritoneal } \\
\text { dialysis patients }\end{array}$ & [263] \\
\hline
\end{tabular}


Table 3. Cont.

\begin{tabular}{|c|c|c|c|c|c|}
\hline & Factor & $\begin{array}{c}\text { Effect on } \\
\text { Hormone Levels }\end{array}$ & $\begin{array}{l}\text { Number of } \\
\text { Participants }\end{array}$ & Participants & Reference \\
\hline & Lead & $\uparrow \mathrm{PTH}$ & 115 & Hemodialysis patients & [264] \\
\hline & Lead & $\leftrightarrow \mathrm{PTH}$ & 47 & Healthy adults & [265] \\
\hline & Lead & $\uparrow \mathrm{PTH}$ & 73 (women) & Healthy adults & {$[266]$} \\
\hline & Lead & $\uparrow \mathrm{iPTH}$ & 93 & Hemodialysis patients & [267] \\
\hline & Uranium & $\leftrightarrow \mathrm{iPTH}$ & 35 & $\begin{array}{l}\text { Gulf War I veterans exposed } \\
\text { to uranium }\end{array}$ & [268] \\
\hline & Uranium & $\downarrow \mathrm{iPTH}$ & 35 & $\begin{array}{l}\text { Gulf War I veterans exposed } \\
\text { to uranium }\end{array}$ & [246] \\
\hline \multirow{11}{*}{ 胥 } & $\begin{array}{c}\text { Persistent } \\
\text { organochlorine } \\
\text { compounds (CB-153) }\end{array}$ & $\leftrightarrow \mathrm{PTH}$ & \multirow[b]{2}{*}{908 (women) } & \multirow[b]{2}{*}{ Healthy adults } & \multirow[b]{2}{*}{ [132] } \\
\hline & $\begin{array}{l}\text { Persistent } \\
\text { organochlorine } \\
\text { compounds } \\
\left(\mathrm{p}, \mathrm{p}^{\prime}-\mathrm{DDE}\right)\end{array}$ & $\leftrightarrow \mathrm{PTH}$ & & & \\
\hline & PFAS & $\uparrow \mathrm{PTH}$ & 100 (men) & Healthy adults & [251] \\
\hline & $\begin{array}{l}\text { PCBs (exposed } \\
\text { prenatally) }\end{array}$ & $\leftrightarrow \mathrm{PTH}$ & 110 & $\begin{array}{l}\text { Children in a mother-child } \\
\text { cohort }\end{array}$ & [250] \\
\hline & Fluoride & $\uparrow \mathrm{PTH}$ & 196 & Healthy adults & [256] \\
\hline & Fluoride & $\uparrow \mathrm{PTH}$ & 84 & $\begin{array}{l}\text { Patients with endemic } \\
\text { fluorosis and healthy } \\
\text { controls }\end{array}$ & [252] \\
\hline & Fluoride & $\begin{array}{c}\downarrow \text { PTH (in pregnant } \\
\text { women) } \\
\leftrightarrow \text { PTH (in new-borns) }\end{array}$ & 180 & $\begin{array}{l}\text { Pregnant women and their } \\
\text { new-borns }\end{array}$ & [269] \\
\hline & Lithium & $\leftrightarrow \mathrm{iPTH}$ & 178 & Mother-child cohort & [270] \\
\hline & Perchlorate & $\downarrow \mathrm{PTH}$ & 2207 (women) & Healthy adults & [19] \\
\hline & Nitrate & $\downarrow \mathrm{PTH}$ & 4265 & Healthy adults & [19] \\
\hline & Thiocyanate & $\downarrow \mathrm{PTH}$ & 4265 & Healthy adults & [19] \\
\hline
\end{tabular}

iPTH, intact parathyroid hormone; PCB, polychlorinated biphenyl; PFAS, perfluoroalkyl substances; $\mathrm{p}, \mathrm{p}^{\prime}$-DDE, $\mathrm{p}, \mathrm{p}^{\prime}$-diphenyldichloroethene; PTH, parathyroid hormone. Decreased $(\downarrow)$, unchanged $(\leftrightarrow)$, increased $(\uparrow)$.

\section{Conclusions}

In this review, we gave an insight into environmental factors that affect the levels of PTH and calcitonin, two hormones that regulate calcium and phosphate homeostasis. We included literature discussing lifestyle factors (smoking, BMI, diet, alcohol, and exercise) and pollutants (heavy metals and chemicals) (Figure 1). In terms of lifestyle factors, most studies have shown a decrease in PTH levels in smokers, a positive correlation between $\mathrm{BMI}$ and PTH, an increase in PTH levels during exercise, and a decrease in PTH levels after vitamin D and calcium intake (Table 1). The results of studies on the impact of alcohol consumption and intake of different types of food and micronutrients (except for vitamin $\mathrm{D}$ and calcium) showed great variability (Table 1). Regarding studies that analyzed the effect of pollutants on PTH levels, the clearest relationship was between PTH and cadmium, with PTH levels decreasing after cadmium exposure (Table 3). While arsenic exposure did not affect PTH levels, lead exposure resulted in increased PTH levels (Table 3). Several studies have investigated the influence of chemicals on PTH levels in humans. Moreover, data on the effect of chemicals and heavy metals on calcitonin levels in humans are scarce, and most of the knowledge, to date, relies on studies in experimental animals. As for the relationship between lifestyle factors and calcitonin, several studies have been conducted on humans and have given great variability in results. The most consistent results were related to smoking (an increase in calcitonin levels was observed in smokers) (Table 2). Given the important role that PTH and calcitonin play in maintaining calcium and phosphate homeostasis in the body, additional studies on the influence of environmental and genetic factors that could affect the levels of these two hormones are extremely important. 
Author Contributions: T.Z. and M.B.L. conceived the review. The first draft of the manuscript was written by M.B.L., I.G., N.P. and T.Z. revised and edited the manuscript critically for important intellectual content. All authors have read and agreed to the published version of the manuscript.

Funding: This work has been supported by the Croatian Science Foundation under the project "Regulation of Thyroid and Parathyroid Function and Blood Calcium Homeostasis" (No. 2593).

Institutional Review Board Statement: Not applicable.

Informed Consent Statement: Not applicable.

Data Availability Statement: Not applicable.

Conflicts of Interest: Authors declare no conflict of interest.

\begin{abstract}
Abbreviations
1,25(OH)2D3, 1 1 ,25-dihydroxyvitamin D; 25(OH)D, 25-hydroxyvitamin D; As, arsenic; BMD, bone mineral density; BMI, body mass index; Ca, calcium; Cd, cadmium; F, fluoride; FGF-23, fibroblast growth factor 23; iPTH, intact parathyroid hormone; $\mathrm{Mg}$, magnesium; $\mathrm{Pb}$, lead; $\mathrm{PCB}$, polychlorinated biphenyl; PFAS, perfluoroalkyl substances; PFOA, perfluoro-octanoic acid; p,p-DDE, p,pdiphenyldichloroethene; PTH, parathyroid hormone; Zn, zinc.
\end{abstract}

\title{
References
}

1. Tebben, P.J.; Kumar, R. The hormonal regulation of calcium metabolism. In Seldin and Geibisch's The Kidney; Elsevier Inc.: Amsterdam, The Netherlands, 2013; Volume 2, pp. 2249-2272, ISBN 9780123814623.

2. Choi, N.W. Kidney and phosphate metabolism. Electrolytes Blood Press. 2008, 6, 77-85. [CrossRef]

3. Gattineni, J.; Friedman, P.A. Regulation of hormone-sensitive renal phosphate transport. Vitam. Horm. 2015, 98, 249-306. [CrossRef] [PubMed]

4. Talmage, R.V.; Vanderwiel, C.J.; Matthews, J.L. Calcitonin and phosphate. Mol. Cell. Endocrinol. 1981, 24, 235-251. [CrossRef]

5. Jafari, N.; Abdollahpour, H.; Falahatkar, B. Stimulatory effects of short-term calcitonin administration on plasma calcium, magnesium, phosphate, and glucose in juvenile Siberian sturgeon Acipenser baerii. Fish Physiol. Biochem. 2020, 46, 1443-1449. [CrossRef] [PubMed]

6. Lofrese, J.J.; Basit, H.; Lappin, S.L. Physiology, Parathyroid; StatPearls Publishing LLC: Treasure Island, FL, USA, 2021.

7. Pearse, A.G. The cytochemistry of the thyroid C cells and their relationship to calcitonin. Proc. R. Soc. Lond. Ser. B Biol. Sci. 1966, 164, 478-487. [CrossRef]

8. Bae, Y.J.; Schaab, M.; Kratzsch, J. Calcitonin as biomarker for the medullary thyroid carcinoma. Recent Res. Cancer Res. 2015, 204, 117-137. [CrossRef]

9. Hunter, D.; De Lange, M.; Snieder, H.; MacGregor, A.J.; Swaminathan, R.; Thakker, R.V.; Spector, T.D. Genetic contribution to bone metabolism, calcium excretion, and vitamin D and parathyroid hormone regulation. J. Bone Miner. Res. 2001, 16, 371-378. [CrossRef] [PubMed]

10. Robinson-Cohen, C.; Lutsey, P.L.; Kleber, M.E.; Nielson, C.M.; Mitchell, B.D.; Bis, J.C.; Eny, K.M.; Portas, L.; Eriksson, J.; Lorentzon, M.; et al. Genetic variants associated with circulating parathyroid hormone. J. Am. Soc. Nephrol. 2017, 28, 1553-1565. [CrossRef]

11. Matana, A.; Brdar, D.; Torlak, V.; Boutin, T.; Popović, M.; Gunjača, I.; Kolčić, I.; Boraska Perica, V.; Punda, A.; Polašek, O.; et al. Genome-wide meta-analysis identifies novel loci associated with parathyroid hormone level. Mol. Med. 2018, 24, 15. [CrossRef]

12. Deftos, L.J.; Weisman, M.H.; Williams, G.W.; Karpf, D.B.; Frumar, A.M.; Davidson, B.J.; Parthemore, J.G.; Judd, H.L. Influence of age and sex on plasma calcitonin in human beings. N. Engl. J. Med. 1980, 302, 1351-1353. [CrossRef]

13. Haden, S.T.; Brown, E.M.; Hurwitz, S.; Scott, J.; Fuleihan, G.E.H. The effects of age and gender on parathyroid hormone dynamics. Clin. Endocrinol. 2000, 52, 329-338. [CrossRef]

14. Carrivick, S.J.; Walsh, J.P.; Brown, S.J.; Wardrop, R.; Hadlow, N.C. Brief report: Does PTH increase with age, independent of 25-hydroxyvitamin D, phosphate, renal function, and ionized calcium? J. Clin. Endocrinol. Metab. 2015, 100, 2131-2134. [CrossRef]

15. Tiegs, R.D.; Body, J.J.; Barta, J.M.; Heath, H. Secretion and metabolism of monomeric human calcitonin: Effects of age, sex, and thyroid damage. J. Bone Miner. Res. 1986, 1, 339-349. [CrossRef]

16. Mazeh, H.; Sippel, R.S.; Chen, H. The role of gender in primary hyperparathyroidism: Same disease, different presentation. Ann. Surg. Oncol. 2012, 19, 2958-2962. [CrossRef] [PubMed]

17. Song, E.; Jeon, M.J.; Yoo, H.J.; Bae, S.J.; Kim, T.Y.; Kim, W.B.; Shong, Y.K.; Kim, H.K.; Kim, W.G. Gender-dependent reference range of serum calcitonin levels in healthy Korean adults. Endocrinol. Metab. 2021, 36, 365-373. [CrossRef]

18. Schutte, R.; Nawrot, T.S.; Richart, T.; Thijs, L.; Vanderschueren, D.; Kuznetsova, T.; Van Hecks, E.; Roels, H.A.; Staessen, J.A. Bone resorption and environmental exposure to cadmium in women: A population study. Environ. Health Perspect. 2008, $116,777$. [CrossRef] [PubMed] 
19. Ko, W.C.; Liu, C.L.; Lee, J.J.; Liu, T.P.; Yang, P.S.; Hsu, Y.C.; Cheng, S.P. Negative association between serum parathyroid hormone levels and urinary perchlorate, nitrate, and thiocyanate concentrations in U.S. adults: The national health and nutrition examination survey 2005-2006. PLoS ONE 2014, 9, e115245. [CrossRef]

20. Soria, M.; Haro, C.G.; Ansón, M.A.; Iñigo, C.; Calvo, M.L.; Escanero, J.F. Variations in serum magnesium and hormonal levels during incremental exercise. Magnes. Res. 2014, 27, 155-164. [CrossRef]

21. Popović, M.; Matana, A.; Torlak, V.; Brdar, D.; Gunjača, I.; Boraska Perica, V.; Barbalić, M.; Kolčić, I.; Punda, A.; Polašek, O.; et al. The effect of multiple nutrients on plasma parathyroid hormone level in healthy individuals. Int. J. Food Sci. Nutr. 2019, 70, 638-644. [CrossRef] [PubMed]

22. Penido, M.G.M.G.; Alon, U.S. Phosphate homeostasis and its role in bone health. Pediatr. Nephrol. 2012, 27, 2039-2048. [CrossRef]

23. Carter, P.; Schipani, E. The roles of parathyroid hormone and calcitonin in bone remodeling: Prospects for novel therapeutics. Endocr. Metab. Immune Disord. Drug Targets 2006, 6, 59-76. [CrossRef]

24. Khundmiri, S.J.; Murray, R.D.; Lederer, E. PTH and Vitamin, D. Compr. Physiol. 2016, 6, 561-601. [CrossRef] [PubMed]

25. Silver, J.; Levi, R. Regulation of PTH synthesis and secretion relevant to the management of secondary hyperparathyroidism in chronic kidney disease. Kidney Int. Suppl. 2005, 67, S8-S12. [CrossRef] [PubMed]

26. Gutiérrez, O.M.; Mannstadt, M.; Isakova, T.; Rauh-Hain, J.A.; Tamez, H.; Shah, A.; Smith, K.; Lee, H.; Thadhani, R.; Jüppner, H.; et al. Fibroblast growth factor 23 and mortality among patients undergoing hemodialysis. N. Engl. J. Med. 2008, 359, 584-592. [CrossRef] [PubMed]

27. Saki, F.; Kassaee, S.R.; Salehifar, A.; Omrani, G.H.R. Interaction between serum FGF-23 and PTH in renal phosphate excretion, a case-control study in hypoparathyroid patients. BMC Nephrol. 2020, 21, 1-6. [CrossRef] [PubMed]

28. Lanske, B.; Razzaque, M.S. Molecular interactions of FGF23 and PTH in phosphate regulation. Kidney Int. 2014, 86, 1072-1074. [CrossRef]

29. Ben-Dov, I.Z.; Galitzer, H.; Lavi-Moshayoff, V.; Goetz, R.; Kuro-o, M.; Mohammadi, M.; Sirkis, R.; Naveh-Many, T.; Silver, J. The parathyroid is a target organ for FGF23 in rats. J. Clin. Investig. 2007, 117, 4003-4008. [CrossRef] [PubMed]

30. Jorde, R.; Saleh, F.; Figenschau, Y.; Kamycheva, E.; Haug, E.; Sundsfjord, J. Serum parathyroid hormone (PTH) levels in smokers and non-smokers. The fifth Tromsø study. Eur. J. Endocrinol. 2005, 152, 39-45. [CrossRef]

31. He, J.L.; Scragg, R.K. Vitamin D, parathyroid hormone, and blood pressure in the National Health and Nutrition Examination Surveys. Am. J. Hypertens. 2011, 24, 911-917. [CrossRef]

32. Paik, J.M.; Farwell, W.R.; Taylor, E.N. Demographic, dietary, and serum factors and parathyroid hormone in the National Health and Nutrition Examination Survey. Osteoporos. Int. 2012, 23, 1727-1736. [CrossRef]

33. Díaz-Gómez, N.M.; Mendoza, C.; González-González, N.L.; Barroso, F.; Jiménez-Sosa, A.; Domenech, E.; Clemente, I.; Barrios, Y.; Moya, M. Maternal smoking and the vitamin D-parathyroid hormone system during the perinatal period. J. Pediatr. 2007, 151, 618-623. [CrossRef] [PubMed]

34. Åkesson, A.; Bjellerup, P.; Lundh, T.; Lidfeldt, J.; Nerbrand, C.; Samsioe, G.; Skerfving, S.; Vahter, M. Cadmium-induced effects on bone in a population-based study of women. Environ. Health Perspect. 2006, 114, 830-834. [CrossRef] [PubMed]

35. Mousavi, S.E.; Amini, H.; Heydarpour, P.; Amini Chermahini, F.; Godderis, L. Air pollution, environmental chemicals, and smoking may trigger vitamin D deficiency: Evidence and potential mechanisms. Environ. Int. 2019, 122, 67-90. [CrossRef] [PubMed]

36. Al-Bashaireh, A.M.; Haddad, L.G.; Weaver, M.; Chengguo, X.; Kelly, D.L.; Yoon, S. The effect of tobacco smoking on bone mass: An overview of pathophysiologic mechanisms. J. Osteoporos. 2018, 2018, 1206235. [CrossRef]

37. Need, A.G.; Kemp, A.; Giles, N.; Morris, H.A.; Horowitz, M.; Nordin, B.E.C. Relationships between intestinal calcium absorption, serum vitamin D metabolites and smoking in postmenopausal women. Osteoporos. Int. 2002, 13, 83-88. [CrossRef]

38. Jorde, R.; Stunes, A.K.; Kubiak, J.; Grimnes, G.; Thorsby, P.M.; Syversen, U. Smoking and other determinants of bone turnover. PLoS ONE 2019, 14, e0225539. [CrossRef]

39. Babić Leko, M.; Gunjača, I.; Pleić, N.; Zemunik, T. Environmental factors affecting thyroid-stimulating hormone and thyroid hormone levels. Int. J. Mol. Sci. 2021, 22, 6521. [CrossRef]

40. Tabassian, A.R.; Nylen, E.S.; Giron, A.E.; Snider, R.H.; Cassidy, M.M.; Becker, K.L. Evidence for cigarette smoke-induced calcitonin secretion from lungs of man and hamster. Life Sci. 1988, 42, 2323-2329. [CrossRef]

41. Yuan, T.J.; Chen, L.P.; Pan, Y.L.; Lu, Y.; Sun, L.H.; Zhao, H.Y.; Wang, W.Q.; Tao, B.; Liu, J.M. An inverted U-shaped relationship between parathyroid hormone and body weight, body mass index, body fat. Endocrine 2021, 72, 844-851. [CrossRef]

42. Wortsman, J.; Matsuoka, L.Y.; Chen, T.C.; Lu, Z.; Holick, M.F. Decreased bioavailability of vitamin D in obesity. Am. J. Clin. Nutr. 2000, 72, 690-693. [CrossRef]

43. Bolland, M.J.; Grey, A.B.; Ames, R.W.; Horne, A.M.; Gamble, G.D.; Reid, I.R. Fat mass is an important predictor of parathyroid hormone levels in postmenopausal women. Bone 2006, 38, 317-321. [CrossRef]

44. Ni, Z.; Smogorzewski, M.; Massry, S.G. Effects of parathyroid hormone on cytosolic calcium of rat adipocytes. Endocrinology 1994, 135, 1837-1844. [CrossRef]

45. McCarty, M.F.; Thomas, C.A. PTH excess may promote weight gain by impeding catecholamine-induced lipolysis-implications for the impact of calcium, vitamin D, and alcohol on body weight. Med. Hypotheses 2003, 61, 535-542. [CrossRef]

46. Rickard, D.J.; Wang, F.L.; Rodriguez-Rojas, A.M.; Wu, Z.; Trice, W.J.; Hoffman, S.J.; Votta, B.; Stroup, G.B.; Kumar, S.; Nuttall, M.E. Intermittent treatment with parathyroid hormone $(\mathrm{PTH})$ as well as a non-peptide small molecule agonist of the PTH1 receptor inhibits adipocyte differentiation in human bone marrow stromal cells. Bone 2006, 39, 1361-1372. [CrossRef] 
47. He, Y.; Liu, R.X.; Zhu, M.T.; Shen, W.B.; Xie, J.; Zhang, Z.Y.; Chen, N.; Shan, C.; Guo, X.-z.; Tao, B.; et al. The browning of white adipose tissue and body weight loss in primary hyperparathyroidism. EBioMedicine 2019, 40, 56-66. [CrossRef]

48. Daniels, G.H.; Hegedüs, L.; Marso, S.P.; Nauck, M.A.; Zinman, B.; Bergenstal, R.M.; Mann, J.F.E.; Derving Karsbøl, J.; Moses, A.C.; Buse, J.B.; et al. LEADER 2: Baseline calcitonin in 9340 people with type 2 diabetes enrolled in the Liraglutide Effect and Action in Diabetes: Evaluation of cardiovascular outcome Results (LEADER) trial: Preliminary observations. Diabetes Obes. Metab. 2015, 17, 477-486. [CrossRef] [PubMed]

49. Mathiesen, D.S.; Lund, A.; Vilsbøll, T.; Knop, F.K.; Bagger, J.I. Amylin and calcitonin: Potential therapeutic strategies to reduce body weight and liver fat. Front. Endocrinol. 2021, 11, 1016. [CrossRef] [PubMed]

50. Calvo, M.S.; Kumar, R.; Heath, H. Elevated secretion and action of serum parathyroid hormone in young adults consuming high phosphorus, low calcium diets assembled from common foods. J. Clin. Endocrinol. Metab. 1988, 66, 823-829. [CrossRef] [PubMed]

51. Kemi, V.E.; Kärkkäinen, M.U.M.; Rita, H.J.; Laaksonen, M.M.L.; Outila, T.A.; Lamberg-Allardt, C.J.E. Low calcium:phosphorus ratio in habitual diets affects serum parathyroid hormone concentration and calcium metabolism in healthy women with adequate calcium intake. Br. J. Nutr. 2010, 103, 561-568. [CrossRef]

52. Lederer, E. Regulation of serum phosphate. J. Physiol. 2014, 592, 3985-3995. [CrossRef] [PubMed]

53. Qin, Z.; Yang, Q.; Liao, R.; Su, B. The association between dietary inflammatory index and parathyroid hormone in adults with/without chronic kidney disease. Front. Nutr. 2021, 8, 364. [CrossRef]

54. Kerstetter, J.E.; Svastisalee, C.M.; Caseria, D.M.; Mitnick, M.A.E.; Insogna, K.L. A threshold for low-protein-diet-induced elevations in parathyroid hormone. Am. J. Clin. Nutr. 2000, 72, 168-173. [CrossRef] [PubMed]

55. Giannini, S.; Nobile, M.; Sartori, L.; Carbonare, L.D.; Ciuffreda, M.; Corrò, P.; D'Angelo, A.; Calò, L.; Crepaldi, G. Acute effects of moderate dietary protein restriction in patients with idiopathic hypercalciuria and calcium nephrolithiasis. Am. J. Clin. Nutr. 1999, 69, 267-271. [CrossRef]

56. Josse, A.R.; Atkinson, S.A.; Tarnopolsky, M.A.; Phillips, S.M. Diets higher in dairy foods and dietary protein support bone health during diet- and exercise-induced weight loss in overweight and obese premenopausal women. J. Clin. Endocrinol. Metab. 2012, 97, 251-260. [CrossRef]

57. Merrill, R.M.; Aldana, S.G. Consequences of a plant-based diet with low dairy consumption on intake of bone-relevant nutrients. J. Women's Health 2009, 18, 691-698. [CrossRef]

58. Hansen, T.H.; Madsen, M.T.B.; Jørgensen, N.R.; Cohen, A.S.; Hansen, T.; Vestergaard, H.; Pedersen, O.; Allin, K.H. Bone turnover calcium homeostasis, and vitamin D status in Danish vegans. Eur. J. Clin. Nutr. 2018, 72, 1046-1054. [CrossRef] [PubMed]

59. Lamberg-Allardt, C.; Kärkkäinen, M.; Seppänen, R.; Biström, H. Low serum 25-hydroxyvitamin D concentrations and secondary hyperparathyroidism in middle-aged white strict vegetarians. Am. J. Clin. Nutr. 1993, 58, 684-689. [CrossRef] [PubMed]

60. Hu, R.; Huang, X.; Huang, J.; Li, Y.; Zhang, C.; Yin, Y.; Chen, Z.; Jin, Y.; Cai, J.; Cui, F. Long- and short-term health effects of pesticide exposure: A cohort study from China. PLoS ONE 2015, 10, e0128766. [CrossRef]

61. Landin-Wilhelmsen, K.; Wilhelmsen, L.; Lappas, G.; Rosén, T.; Lindstedt, G.; Lundberg, P.A.; Wilske, J.; Bengtsson, B.Å. Serum intact parathyroid hormone in a random population sample of men and women: Relationship to anthropometry, life-style factors, blood pressure, and vitamin D. Calcif. Tissue Int. 1995, 56, 104-108. [CrossRef]

62. Brot, C.; Jøorgensen, N.R.; Sørensen, O.H. The influence of smoking on vitamin D status and calcium metabolism. Eur. J. Clin. Nutr. 1999, 53, 920-926. [CrossRef]

63. Paik, J.M.; Curhan, G.C.; Forman, J.P.; Taylor, E.N. Determinants of plasma parathyroid hormone levels in young women. Calcif. Tissue Int. 2010, 87, 211-217. [CrossRef]

64. Parthemore, J.G.; Deftos, L.J. Calcitonin secretion in normal human subjects. J. Clin. Endocrinol. Metab. 1978, 47, 184-188. [CrossRef]

65. Pedrazzoni, M.; Ciotti, G.; Davoli, L.; Pioli, G.; Girasole, G.; Palummeri, E.; Passeri, M. Meal-stimulated gastrin release and calcitonin secretion. J. Endocrinol. Investig. 1989, 12, 409-412. [CrossRef]

66. Zayed, A.; Alzubaidi, M.; Atallah, S.; Momani, M.; Al-Delaimy, W. Should food intake and circadian rhythm be considered when measuring serum calcitonin level? Endocr. Pract. 2013, 19, 620-626. [CrossRef]

67. Pointillart, A.; Garel, J.M.; Gueguen, L.; Colin, C. Plasma calcitonin and parathyroid hormone levels in growing pigs on different diets. I.-High phosphorus diet. Ann. Biol. Anim. Biochim. Biophys. 1978, 18, 699-709. [CrossRef]

68. Deftos, L.J.; Miller, M.M.; Burton, D.W. A high-fat diet increases calcitonin secretion in the rat. Bone Miner. 1989, 5, 303-308. [CrossRef]

69. Grundmann, M.; von Versen-Höynck, F. Vitamin D-Roles in women's reproductive health? Reprod. Biol. Endocrinol. 2011, 9, 146. [CrossRef] [PubMed]

70. Moslehi, N.; Shab-Bidar, S.; Mirmiran, P.; Hosseinpanah, F.; Azizi, F. Determinants of parathyroid hormone response to vitamin D supplementation: A systematic review and meta-analysis of randomised controlled trials. Br. J. Nutr. 2015, 114, 1360-1374. [CrossRef] [PubMed]

71. Vaidya, A.; Curhan, G.C.; Paik, J.M.; Wang, M.; Taylor, E.N. Physical activity and the risk of primary hyperparathyroidism. J. Clin. Endocrinol. Metab. 2016, 101, 1590-1597. [CrossRef] [PubMed]

72. Engström, A.; Håkansson, H.; Skerfving, S.; Bjellerup, P.; Lidfeldt, J.; Lundh, T.; Samsioe, G.; Vahter, M.; Åkesson, A. Retinol may counteract the negative effect of cadmium on bone. J. Nutr. 2011, 141, 2198-2203. [CrossRef] [PubMed]

73. Liu, W.; Ridefelt, P.; Akerström, G.; Hellman, P. Differentiation of human parathyroid cells in culture. J. Endocrinol. 2001, 168, 417-425. [CrossRef] 
74. MacDonald, P.N.; Ritter, C.; Brown, A.J.; Slatopolsky, E. Retinoic acid suppresses parathyroid hormone (PTH) secretion and PreproPTH mRNA levels in bovine parathyroid cell culture. J. Clin. Investig. 1994, 93, 725-730. [CrossRef] [PubMed]

75. Ooms, M.E.; Roos, J.C.; Bezemer, P.D.; van der Vijgh, W.J.F.; Bouter, L.M.; Lips, P. Prevention of bone loss by vitamin D supplementation in elderly women: A randomized double-blind trial. J. Clin. Endocrinol. Metab. 1995, 80, 1052-1058. [CrossRef]

76. Naveh-Many, T.; Silver, J. Regulation of calcitonin gene transcription by vitamin D metabolites in vivo in the rat. J. Clin. Investig. 1988, 81, 270-273. [CrossRef]

77. Saggese, G.; Federico, G.; Bertelloni, S.; Baroncelli, G.I.; Calisti, L. Hypomagnesemia and the parathyroid hormone-vitamin D endocrine system in children with insulin-dependent diabetes mellitus: Effects of magnesium administration. J. Pediatr. 1991, 118, 220-225. [CrossRef]

78. Sahota, O.; Mundey, M.K.; San, P.; Godber, I.M.; Hosking, D.J. Vitamin D insufficiency and the blunted PTH response in established osteoporosis: The role of magnesium deficiency. Osteoporos. Int. 2006, 17, 1013-1021. [CrossRef]

79. Cheung, M.M.; DeLuccia, R.; Ramadoss, R.K.; Aljahdali, A.; Volpe, S.L.; Shewokis, P.A.; Sukumar, D. Low dietary magnesium intake alters vitamin D-parathyroid hormone relationship in adults who are overweight or obese. Nutr. Res. 2019, 69, 82-93. [CrossRef] [PubMed]

80. Dai, L.J.; Ritchie, G.; Kerstan, D.; Kang, H.S.; Cole, D.E.C.; Quamme, G.A. Magnesium transport in the renal distal convoluted tubule. Physiol. Rev. 2001, 81, 51-84. [CrossRef]

81. McGonigle, R.; Weston, M.; Keenan, J.; Jackson, D.; Parsons, V. Effect of hypermagnesemia on circulating plasma parathyroid hormone in patients on regular hemodialysis therapy. Magnesium 1984, 3, 1-7. [PubMed]

82. Ohya, M.; Negi, S.; Sakaguchi, T.; Koiwa, F.; Ando, R.; Komatsu, Y.; Shinoda, T.; Inaguma, D.; Joki, N.; Yamaka, T.; et al. Significance of serum magnesium as an independent correlative factor on the parathyroid hormone level in uremic patients. J. Clin. Endocrinol. Metab. 2014, 99, 3873-3878. [CrossRef]

83. Nishiyama, S.; Nakamura, T.; Higashi, A.; Matsuda, I. Infusion of zinc inhibits serum calcitonin levels in patients with various zinc status. Calcif. Tissue Int. 1991, 49, 179-182. [CrossRef]

84. Suzuki, T.; Kajita, Y.; Katsumata, S.I.; Matsuzaki, H.; Suzuki, K. Zinc deficiency increases serum concentrations of parathyroid hormone through a decrease in serum calcium and induces bone fragility in rats. J. Nutr. Sci. Vitaminol. 2015, 61, 382-390. [CrossRef]

85. Alkan Baylan, F.; Bankir, M.; Acıbucu, F.; Kılınç, M. Zinc copper levels in patients with primary hyperparathyroidism. Cumhur. Med. J. 2021, 43, 117-123. [CrossRef]

86. Nielsen, F.H.; Milne, D.B. A moderately high intake compared to a low intake of zinc depresses magnesium balance and alters indices of bone turnover in postmenopausal women. Eur. J. Clin. Nutr. 2004, 58, 703-710. [CrossRef]

87. Strause, L.; Saltman, P.; Smith, K.T.; Bracker, M.; Andon, M.B. Spinal bone loss in postmenopausal women supplemented with calcium and trace minerals. J. Nutr. 1994, 124, 1060-1064. [CrossRef]

88. Eaton Evans, J.; Mcilrath, E.M.; Jackson, W.E.; Mccartney, H.; Strain, J.J. Copper supplementation and the maintenance of bone mineral density in middle-aged women. J. Trace Elem. Exp. Med. 1996, 9, 87-94. [CrossRef]

89. Marrone, J.A.; Maddalozzo, G.F.; Branscum, A.J.; Hardin, K.; Cialdella-Kam, L.; Philbrick, K.A.; Breggia, A.C.; Rosen, C.J.; Turner, R.T.; Iwaniec, U.T. Moderate alcohol intake lowers biochemical markers of bone turnover in postmenopausal women. Menopause 2012, 19, 974-979. [CrossRef] [PubMed]

90. Laitinen, K.; Lamberg-Allardt, C.; Tunninen, R.; Karonen, S.-L.; Tähtelä, R.; Ylikahri, R.; Välimäki, M. Transient hypoparathyroidism during acute alcohol intoxication. N. Engl. J. Med. 1991, 324, 721-727. [CrossRef] [PubMed]

91. Rapuri, P.B.; Gallagher, J.C.; Balhorn, K.E.; Ryschon, K.L. Smoking and bone metabolism in elderly women. Bone 2000, 27, 429-436. [CrossRef]

92. Ilich, J.Z.; Brownbill, R.A.; Tamborini, L.; Crncevic-Orlic, Z. To drink or not to drink: How are alcohol, caffeine and past smoking related to bone mineral density in elderly women? J. Am. Coll. Nutr. 2002, 21, 536-544. [CrossRef]

93. Rico, H. Alcohol and bone disease. Alcohol Alcohol. 1990, 25, 345-352. [PubMed]

94. Vantyghem, M.C.; Danel, T.; Marcelli-Tourvieille, S.; Moriau, J.; Leclerc, L.; Cardot-Bauters, C.; Docao, C.; Carnaille, B.; Wemeau, J.L.; D'Herbomez, M. Calcitonin levels do not decrease with weaning in chronic alcoholism. Thyroid 2007, 17, $213-217$. [CrossRef] [PubMed]

95. Schuster, R.; Koopmann, A.; Grosshans, M.; Reinhard, I.; Spanagel, R.; Kiefer, F. Association of plasma calcium concentrations with alcohol craving: New data on potential pathways. Eur. Neuropsychopharmacol. 2017, 27, 42-47. [CrossRef] [PubMed]

96. Ilias, I.; Paparrigopoulos, T.; Tzavellas, E.; Karaiskos, D.; Meristoudis, G.; Liappas, A.; Liappas, I. Inpatient alcohol detoxification and plasma calcitonin (with original findings). Hell. J. Nucl. Med. 2011, 14, 177-178.

97. Kalafateli, A.L.; Vallöf, D.; Colombo, G.; Lorrai, I.; Maccioni, P.; Jerlhag, E. An amylin analogue attenuates alcohol-related behaviours in various animal models of alcohol use disorder. Neuropsychopharmacology 2019, 44, 1093-1102. [CrossRef]

98. Kalafateli, A.L.; Satir, T.M.; Vallöf, D.; Zetterberg, H.; Jerlhag, E. An amylin and calcitonin receptor agonist modulates alcohol behaviors by acting on reward-related areas in the brain. Prog. Neurobiol. 2021, 200, 101969. [CrossRef]

99. Ylli, D.; Wartofsky, L. Can we link thyroid status, energy expenditure, and body composition to management of subclinical thyroid dysfunction? J. Clin. Endocrinol. Metab. 2019, 104, 209-212. [CrossRef]

100. Bouassida, A.; Latiri, I.; Bouassida, S.; Zalleg, D.; Zaouali, M.; Feki, Y.; Gharbi, N.; Zbidi, A.; Tabka, Z. Parathyroid hormone and physical exercise: A brief review. J. Sport. Sci. Med. 2006, 5, 367. 
101. Nichols, J.F.; Palmer, J.E.; Levy, S.S. Low bone mineral density in highly trained male master cyclists. Osteoporos. Int. 2003, 14, 644-649. [CrossRef] [PubMed]

102. Hind, K.; Truscott, J.G.; Evans, J.A. Low lumbar spine bone mineral density in both male and female endurance runners. Bone 2006, 39, 880-885. [CrossRef]

103. Lombardi, G.; Ziemann, E.; Banfi, G.; Corbetta, S. Physical activity-dependent regulation of parathyroid hormone and calciumphosphorous metabolism. Int. J. Mol. Sci. 2020, 21, 5388. [CrossRef]

104. Shea, K.L.; Barry, D.W.; Sherk, V.D.; Hansen, K.C.; Wolfe, P.; Kohrt, W.M. Calcium supplementation and parathyroid hormone response to vigorous walking in postmenopausal women. Med. Sci. Sport. Exerc. 2014, 46, 2007-2013. [CrossRef]

105. Blum, J.W.; Fischer, J.A.; Hunziker, W.H.; Binswanger, U.; Picotti, G.B.; Da Prada, M.; Guillebeau, A. Parathyroid hormone responses to catecholamines and to changes of extracellular calcium in cows. J. Clin. Investig. 1978, 61, 1113-1122. [CrossRef] [PubMed]

106. López, I.; Aguilera-Tejero, E.; Estepa, J.C.; Rodríguez, M.; Felsenfeld, A.J. Role of acidosis-induced increases in calcium on PTH secretion in acute metabolic and respiratory acidosis in the dog. Am. J. Physiol. Endocrinol. Metab. 2004, 286, E780-E785. [CrossRef]

107. Lin, L.L.; Hsieh, S.S. Effects of strength and endurance exercise on calcium-regulating hormones between different levels of physical activity. J. Mech. Med. Biol. 2005, 5, 267-275. [CrossRef]

108. Zhao, C.; Hou, H.; Chen, Y.; Lv, K. Effect of aerobic exercise and raloxifene combination therapy on senileosteoporosis. J. Phys. Ther. Sci. 2016, 28, 1791. [CrossRef] [PubMed]

109. Henderson, S.A.; Graham, H.K.; Mollan, R.A.B.; Riddoch, C.; Sheridan, B.; Johnston, H. Calcium homeostasis and exercise. Int Orthop. 1989, 13, 69-73. [CrossRef]

110. O'Neill, M.E.; Wilkinson, M.; Robinson, B.G.; McDowall, D.B.; Cooper, K.A.; Mihailidou, A.S.; Frewin, D.B.; Clifton-Bligh, P.; Hunyor S.N. The effect of exercise on circulating immunoreactive calcitonin in men. Horm. Metab. Res. 1990, 22, 546-550. [CrossRef]

111. Klausen, T.; Breum, L.; Sørensen, H.A.; Schifter, S.; Sonne, B. Plasma levels of parathyroid hormone, vitamin D, calcitonin, and calcium in association with endurance exercise. Calcif. Tissue Int. 1993, 52, 205-208. [CrossRef] [PubMed]

112. Soria, M.; Anson, M.; Escanero, J.F. Correlation analysis of exercise-induced changes in plasma trace element and hormone levels during incremental exercise in well-trained athletes. Biol. Trace Elem. Res. 2016, 170, 55-64. [CrossRef]

113. Çetin Kargin, N.; Marakoglu, K.; Unlu, A.; Kebapcilar, L.; Korucu, N. Comparison of bone turnover markers between male smoker and non-smoker. Acta Med. Mediterr. 2016, 32, 317-323. [CrossRef]

114. Fujiyoshi, A.; Polgreen, L.E.; Gross, M.D.; Reis, J.P.; Sidney, S.; Jacobs, D.R. Smoking habits and parathyroid hormone concentrations in young adults: The CARDIA study. Bone Rep. 2016, 5, 104. [CrossRef]

115. Andersen, S.; Noahsen, P.; Rex, K.F.; Fleischer, I.; Albertsen, N.; Jorgensen, M.E.; Schæbel, L.K.; Laursen, M.B. Serum 25hydroxyvitamin D, calcium and parathyroid hormone levels in Native and European populations in Greenland. Br. J. Nutr. 2018, 119, 391-397. [CrossRef] [PubMed]

116. Chen, W.R.; Sha, Y.; Chen, Y.D.; Shi, Y.; Yin, D.W.; Wang, H. Vitamin D, parathyroid hormone, and serum lipid profiles in a middle-aged and elderly Chinese population. Endocr. Pract. 2014, 20, 556-565. [CrossRef] [PubMed]

117. Cutillas-Marco, E.; Fuertes-Prosper, A.; Grant, W.B.; Morales-Suárez-Varela, M. Vitamin D deficiency in South Europe: Effect of smoking and aging. Photodermatol. Photoimmunol. Photomed. 2012, 28, 159-161. [CrossRef] [PubMed]

118. Supervía, A.; Nogués, X.; Enjuanes, A.; Vila, J.; Mellibovsky, L.; Serrano, S.; Aubía, J.; Díez-Pérez, A. Effect of smoking and smoking cessation on bone mass, bone remodeling, vitamin D, PTH and sex hormones. J. Musculoskelet. Neuronal Interact. 2006, 6, 234-241.

119. Hagström, E.; Hellman, P.; Larsson, T.E.; Ingelsson, E.; Berglund, L.; Sundström, J.; Melhus, H.; Held, C.; Lind, L.; Michaëlsson, K.; et al. Plasma parathyroid hormone and the risk of cardiovascular mortality in the community. Circulation 2009, 119, $2765-2771$. [CrossRef] [PubMed]

120. Kamycheva, E.; Sundsfjord, J.; Jorde, R. Serum parathyroid hormone levels predict coronary heart disease: The Tromsø Study. Eur. J. Cardiovasc. Prev. Rehabil. 2004, 11, 69-74. [CrossRef]

121. Li, L.; Yin, X.; Yao, C.; Zhu, X.; Wu, X. Vitamin D, parathyroid hormone and their associations with hypertension in a Chinese population. PLoS ONE 2012, 7, e43344. [CrossRef]

122. Lorentzon, M.; Mellström, D.; Haug, E.; Ohlsson, C. Smoking is associated with lower bone mineral density and reduced cortical thickness in young men. J. Clin. Endocrinol. Metab. 2007, 92, 497-503. [CrossRef]

123. Mellstrom, D.; Johansson, C.; Johnell, O.; Lindstedt, G.; Lundberg, P.A.; Obrant, K.; Schoon, I.M.; Toss, G.; Ytterberg, B.O. Osteoporosis, metabolic aberrations, and increased risk for vertebral fractures after partial gastrectomy. Calcif. Tissue Int. 1993, 53, 370-377. [CrossRef] [PubMed]

124. Muntner, P.; Jones, T.M.; Hyre, A.D.; Melamed, M.L.; Alper, A.; Raggi, P.; Leonard, M.B. Association of serum intact parathyroid hormone with lower estimated glomerular filtration rate. Clin. J. Am. Soc. Nephrol. 2009, 4, 186-194. [CrossRef] [PubMed]

125. Ortego-Centeno, N.; Muñoz-Torres, M.; Jódar, E.; Hernández-Quero, J.; Jurado-Duce, A.; De La Higuera Torres-Puchol, J. Effect of tobacco consumption on bone mineral density in healthy young males. Calcif. Tissue Int. 1997, 60, 496-500. [CrossRef]

126. Saquib, N.; Von Mühlen, D.; Garland, C.F.; Barrett-Connor, E. Serum 25-hydroxyvitamin D, parathyroid hormone, and bone mineral density in men: The Rancho Bernardo study. Osteoporos. Int. 2006, 17, 1734-1741. [CrossRef]

127. Sneve, M.; Emaus, N.; Joakimsen, R.M.; Jorde, R. The association between serum parathyroid hormone and bone mineral density, and the impact of smoking: The Tromso Study. Eur. J. Endocrinol. 2008, 158, 401-409. [CrossRef] [PubMed] 
128. Snijder, M.B.; Lips, P.; Seidell, J.C.; Visser, M.; Deeg, D.J.H.; Dekker, J.M.; Van Dam, R.M. Vitamin D status and parathyroid hormone levels in relation to blood pressure: A population-based study in older men and women. J. Intern. Med. 2007, 261, 558-565. [CrossRef]

129. Szulc, P.; Garnero, P.; Claustrat, B.; Marchand, F.; Duboeuf, F.; Delmas, P.D. Increased bone resorption in moderate smokers with low body weight: The Minos study. J. Clin. Endocrinol. Metab. 2002, 87, 666-674. [CrossRef] [PubMed]

130. Trevisan, C.; Alessi, A.; Girotti, G.; Zanforlini, B.M.; Bertocco, A.; Mazzochin, M.; Zoccarato, F.; Piovesan, F.; Dianin, M.; Giannini, S.; et al. The impact of smoking on bone metabolism, bone mineral density and vertebral fractures in postmenopausal women. J. Clin. Densitom. 2020, 23, 381-389. [CrossRef]

131. Gudmundsson, J.A.; Ljunghall, S.; Bergquist, C.; Wide, L.; Nillius, S.J. Increased bone turnover during gonadotropin-releasing hormone superagonist-induced ovulation inhibition. J. Clin. Endocrinol. Metab. 1987, 65, 159-163. [CrossRef]

132. Rignell-Hydbom, A.; Skerfving, S.; Lundh, T.; Lindh, C.H.; Elmståhl, S.; Bjellerup, P.; Jünsson, B.A.; Strümberg, U.; Akesson, A. Exposure to cadmium and persistent organochlorine pollutants and its association with bone mineral density and markers of bone metabolism on postmenopausal women. Environ. Res. 2009, 109, 991-996. [CrossRef]

133. Eliasson, M.; Hagg, E.; Lundblad, D.; Karlsson, R.; Bucht, E. Influence of smoking and snuff use on electrolytes, adrenal and calcium regulating hormones. Acta Endocrinol. 1993, 128, 35-40. [CrossRef] [PubMed]

134. Bikle, D.D.; Stesin, A.; Halloran, B.; Steinbach, L.; Recker, R. Alcohol-induced bone disease: Relationship to age and parathyroid hormone levels. Alcohol. Clin. Exp. Res. 1993, 17, 690-695. [CrossRef]

135. Johnell, O.; Kristensson, H.; Nilsson, B.E. Parathyroid activity in alcoholics. Br. J. Addict. 1982, 77, 93-95. [CrossRef]

136. Sripanyakorn, S.; Jugdaohsingh, R.; Mander, A.; Davidson, S.L.; Thompson, R.P.H.; Powell, J.J. Moderate ingestion of alcohol is associated with acute ethanol-induced suppression of circulating CTX in a PTH-independent fashion. J. Bone Miner. Res. 2009, 24, 1380-1388. [CrossRef]

137. Wilkens Knudsen, A.; Jensen, J.E.B.; Nordgaard-Lassen, I.; Almdal, T.; Kondrup, J.; Becker, U. Nutritional intake and status in persons with alcohol dependency: Data from an outpatient treatment programme. Eur. J. Nutr. 2014, 53, 1483-1492. [CrossRef] [PubMed]

138. Kim, M.J.; Shim, M.S.; Kim, M.K.; Lee, Y.; Shin, Y.G.; Chung, C.H.; Kwon, S.O. Effect of chronic alcohol ingestion on bone mineral density in males without liver cirrhosis. Korean J. Intern. Med. 2003, 18, 174-180. [CrossRef] [PubMed]

139. Perry, H.; Horowitz, M.; Fleming, S.; Kaiser, F.; Patrick, P.; Morley, J.; Cushman, W.; Bingham, S.; Perry, H. Effect of recent alcohol intake on parathyroid hormone and mineral metabolism in men. Alcohol. Clin. Exp. Res. 1998, 22, 1369-1375. [CrossRef]

140. Al-Sultan, A.; Amin, T.; Abou-Seif, M.; Naboli, M. Al Vitamin D, parathyroid hormone levels and insulin sensitivity among obese young adult Saudis. Eur. Rev. Med. Pharmacol. Sci. 2011, 15, 135-147.

141. Bischof, M.G.; Heinze, G.; Vierhapper, H. Vitamin D status and its relation to age and body mass index. Horm. Res. 2006, 66, 211-215. [CrossRef]

142. Di Monaco, M.; Castiglioni, C.; Vallero, F.; Di Monaco, R.; Tappero, R. Parathyroid hormone is significantly associated with body fat compartment in men but not in women following a hip fracture. Aging Clin. Exp. Res. 2013, 25, 371-376. [CrossRef] [PubMed]

143. Drechsler, C.; Grootendorst, D.C.; Boeschoten, E.W.; Krediet, R.T.; Wanner, C.; Dekker, F.W. Changes in parathyroid hormone, body mass index and the association with mortality in dialysis patients. Nephrol. Dial. Transplant. 2011, 26, 1340-1346. [CrossRef]

144. Durá-Travé, T.; Gallinas-Victoriano, F.; Chueca-Guindulain, M.J.; Berrade-Zubiri, S.; Urretavizcaya-Martinez, M.; AhmedMohamed, L. Assessment of vitamin D status and parathyroid hormone during a combined intervention for the treatment of childhood obesity. Nutr. Diabetes 2019, 9, 1-18. [CrossRef]

145. Abbasalizad Farhangi, M.; Ostadrahimi, A.; Mahboob, S. Serum calcium, magnesium, phosphorous and lipid profile in healthy Iranian premenopausal women. Biochem. Med. 2011, 21, 312-320. [CrossRef]

146. Gannagé-Yared, M.H.; Chemali, R.; Sfeir, C.; Maalouf, G.; Halaby, G. Dietary calcium and vitamin D intake in an adult Middle Eastern population: Food sources and relation to lifestyle and PTH. Int. J. Vitam. Nutr. Res. 2005, 75, 281-289. [CrossRef] [PubMed]

147. Garcia, V.C.; Schuch, N.J.; Catania, A.S.; Gouvea Ferreira, S.R.; Martini, L.A. Parathyroid hormone has an important role in blood pressure regulation in vitamin D-insufficient individuals. Nutrition 2013, 29, 1147-1151. [CrossRef]

148. Grethen, E.; Hill, K.M.; Jones, R.; Cacucci, B.M.; Gupta, C.E.; Acton, A.; Considine, R.V.; Peacock, M. Serum leptin, parathyroid hormone, 1,25-dihydroxyvitamin D, fibroblast growth factor 23, bone alkaline phosphatase, and sclerostin relationships in obesity. J. Clin. Endocrinol. Metab. 2012, 97, 1655-1662. [CrossRef] [PubMed]

149. Guasch, A.; Bulló, M.; Rabassa, A.; Bonada, A.; Del Castillo, D.; Sabench, F.; Salas-Salvadó, J. Plasma vitamin D and parathormone are associated with obesity and atherogenic dyslipidemia: A cross-sectional study. Cardiovasc. Diabetol. 2012, 11, 1-11. [CrossRef] [PubMed]

150. Guglielmi, V.; Bellia, A.; Gentileschi, P.; Lombardo, M.; D’Adamo, M.; Lauro, D.; Sbraccia, P. Parathyroid hormone in surgery-induced weight loss: No glucometabolic effects but potential adaptive response to skeletal loading. Endocrine 2018, 59, 288-295. [CrossRef]

151. Gunnarsson, Ö.; Indridason, Ó.S.; Franzson, L.; Sigurdsson, G. Factors associated with elevated or blunted PTH response in vitamin D insufficient adults. J. Intern. Med. 2009, 265, 488-495. [CrossRef]

152. Ha, J.; Jo, K.; Lim, D.J.; Lee, J.M.; Chang, S.A.; Kang, M.I.L.; Cha, B.Y.; Kim, M.H. Parathyroid hormone and vitamin D are associated with the risk of metabolic obesity in a middle-aged and older Korean population with preserved renal function: A cross-sectional study. PLoS ONE 2017, 12, e0175132. [CrossRef] 
153. Helal, O.; Kensara, O.; Azzeh, F.; Abdel Kafy, M. Effect of parathyroid hormone and body mass index on overall stability index in Saudi males with vitamin D deficiency. Life Sci. J. 2016, 13, 1-6.

154. Ishimura, E.; Okuno, S.; Tsuboniwa, N.; Norimine, K.; Fukumoto, S.; Yamakawa, K.; Yamakawa, T.; Shoji, S.; Nishizawa, Y.; Inaba, M. Significant positive association between parathyroid hormone and fat mass and lean mass in chronic hemodialysis patients. J. Clin. Endocrinol. Metab. 2013, 98, 1264-1270. [CrossRef] [PubMed]

155. Kamycheva, E.; Sundsfjord, J.; Jorde, R. Serum parathyroid hormone level is associated with body mass index. The 5th Troms $\varnothing$ study. Eur. J. Endocrinol. 2004, 151, 167-172. [CrossRef]

156. Kim, H.; Chandler, P.; Ng, K.; Manson, J.A.E.; Giovannucci, E. Obesity and efficacy of vitamin D 3 supplementation in healthy black adults. Cancer Causes Control 2020, 31, 303-307. [CrossRef]

157. Kontogeorgos, G.; Trimpou, P.; Laine, C.M.; Oleröd, G.; Lindahl, A.; Landin-Wilhelmsen, K. Normocalcaemic, vitamin D-sufficient hyperparathyroidism-High prevalence and low morbidity in the general population: A long-term follow-up study, the WHO MONICA project, Gothenburg, Sweden. Clin. Endocrinol. 2015, 83, 277-284. [CrossRef] [PubMed]

158. Kovesdy, C.P.; Ahmadzadeh, S.; Anderson, J.E.; Kalantar-Zadeh, K. Obesity is associated with secondary hyperparathyroidism in men with moderate and severe chronic kidney disease. Clin. J. Am. Soc. Nephrol. 2007, 2, 1024-1029. [CrossRef] [PubMed]

159. Li, M.; Lv, F.; Zhang, Z.; Deng, W.; Li, Y.; Deng, Z.; Jiang, Y.; Wang, O.; Xing, X.; Xu, L.; et al. Establishment of a normal reference value of parathyroid hormone in a large healthy Chinese population and evaluation of its relation to bone turnover and bone mineral density. Osteoporos. Int. 2016, 27, 1907-1916. [CrossRef]

160. Marwaha, R.K.; Garg, M.K.; Mahalle, N.; Bhadra, K.; Tandon, N. Role of parathyroid hormone in determination of fat mass in patients with Vitamin D deficiency. Indian J. Endocrinol. Metab. 2017, 21, 848. [CrossRef] [PubMed]

161. Reinehr, T.; de Sousa, G.; Alexy, U.; Kersting, M.; Andler, W. Vitamin D status and parathyroid hormone in obese children before and after weight loss. Eur. J. Endocrinol. 2007, 157, 225-232. [CrossRef]

162. Van Ballegooijen, A.J.; Kestenbaum, B.; Sachs, M.C.; De Boer, I.H.; Siscovick, D.S.; Hoofnagle, A.N.; Ix, J.H.; Visser, M.; Brouwer, I.A. Association of 25-hydroxyvitamin D and parathyroid hormone with incident hypertension: MESA (Multi-Ethnic Study of Atherosclerosis). J. Am. Coll. Cardiol. 2014, 63, 1214-1222. [CrossRef]

163. You, L.; Chrn, L.; Pan, L.; Chen, J.; Peng, Y. Relation of body mass index to vitamin D, PTH, and bone turnover markers levels among women in Shanghai area. Chin. J. Endocrinol. Metab. 2013, 12, 566-569. [CrossRef]

164. Shapses, S.A.; Lee, E.J.; Sukumar, D.; Durazo-Arvizu, R.; Schneider, S.H. The effect of obesity on the relationship between serum parathyroid hormone and 25-hydroxyvitamin D in women. J. Clin. Endocrinol. Metab. 2013, 98, E886-E890. [CrossRef]

165. Shin, S.-G.; Park, S.-K.; Kim, K.-M.; Kim, D.-H. Association of serum parathyroid hormone and vitamin D levels with cardiovascular risk factors. Korean J. Fam. Pract. 2017, 7, 55-59. [CrossRef]

166. Snijder, M.B.; Van Dam, R.M.; Visser, M.; Deeg, D.J.H.; Dekker, J.M.; Bouter, L.M.; Seidell, J.C.; Lips, P. Adiposity in relation to vitamin D status and parathyroid hormone levels: A population-based study in older men and women. J. Clin. Endocrinol. Metab. 2005, 90, 4119-4123. [CrossRef]

167. Svedlund, A.; Pettersson, C.; Tubic, B.; Magnusson, P.; Swolin-Eide, D. Vitamin D status in young Swedish women with anorexia nervosa during intensive weight gain therapy. Eur. J. Nutr. 2017, 56, 2061-2067. [CrossRef]

168. Valiña-Tóth, A.L.B.; Lai, Z.; Yoo, W.; Abou-Samra, A.; Gadegbeku, C.A.; Flack, J.M. Relationship of vitamin D and parathyroid hormone to obesity and body composition in African Americans. Clin. Endocrinol. 2010, 72, 595. [CrossRef] [PubMed]

169. Cardoso, C.K.d.S.; Santos, A.S.e.A.d.C.; Rosa, L.P.d.S.; Mendonça, C.R.; Vitorino, P.V.d.O.; Peixoto, M.D.R.G.; Silveira, É.A. Effect of extra virgin olive oil and traditional Brazilian diet on the bone health parameters of severely obese adults: A randomized controlled trial. Nutrients 2020, 12, 403. [CrossRef]

170. Hassoon, A.; Michos, E.D.; Miller, E.R.; Crisp, Z.; Appel, L.J. Effects of different dietary interventions on calcitriol, parathyroid hormone, calcium, and phosphorus: Results from the DASH trial. Nutrients 2018, 10, 367. [CrossRef]

171. Ho-Pham, L.T.; Vu, B.Q.; Lai, T.Q.; Nguyen, N.D.; Nguyen, T.V. Vegetarianism, bone loss, fracture and vitamin D: A longitudinal study in Asian vegans and non-vegans. Eur. J. Clin. Nutr. 2012, 66, 75-82. [CrossRef] [PubMed]

172. Tylavsky, F.A.; Holliday, K.; Danish, R.; Womack, C.; Norwood, J.; Carbone, L. Fruit and vegetable intakes are an independent predictor of bone size in early pubertal children. Am. J. Clin. Nutr. 2004, 79, 311-317. [CrossRef]

173. Kinyamu, H.K.; Gallagher, J.C.; Rafferty, K.A.; Balhorn, K.E. Dietary calcium and vitamin D intake in elderly women: Effect on serum parathyroid hormone and vitamin D metabolites. Am. J. Clin. Nutr. 1998, 67, 342-348. [CrossRef] [PubMed]

174. Chapuy, M.C.; Chapuy, P.; Meunier, P.J. Calcium and vitamin D supplements: Effects on calcium metabolism in elderly people. Am. J. Clin. Nutr. 1987, 46, 324-328. [CrossRef] [PubMed]

175. Dawson-Hughes, B.; Dallal, G.E.; Krall, E.A.; Harris, S.; Sokoll, L.J.; Falconer, G. Effect of vitamin D supplementation on wintertime and overall bone loss in healthy postmenopausal women. Ann. Intern. Med. 1991, 115, 505-512. [CrossRef]

176. Chapuy, M.C.; Arlot, M.E.; Duboeuf, F.; Brun, J.; Crouzet, B.; Arnaud, S.; Delmas, P.D.; Meunier, P.J. Vitamin D3 and calcium to prevent hip fractures in elderly women. N. Engl. J. Med. 1992, 327, 1637-1642. [CrossRef]

177. Dawson-Hughes, B.; Harris, S.S.; Krall, E.A.; Dallal, G.E. Effect of calcium and vitamin D supplementation on bone density in men and women 65 years of age or older. N. Engl. J. Med. 1997, 337, 670-676. [CrossRef]

178. Krieg, M.A.; Jacquet, A.F.; Bremgartner, M.; Cuttelod, S.; Thiébaud, D.; Burckhardt, P. Effect of supplementation with vitamin D3 and calcium on quantitative ultrasound of bone in elderly institutionalized women: A longitudinal study. Osteoporos. Int. 1999, 9, 483-488. [CrossRef] 
179. Hunter, D.; Major, P.; Arden, N.; Swaminathan, R.; Andrew, T.; MacGregor, A.J.; Keen, R.; Snieder, H.; Spector, T.D. A randomized controlled trial of vitamin D supplementation on preventing postmenopausal bone loss and modifying bone metabolism using identical twin pairs. J. Bone Miner. Res. 2000, 15, 2276-2283. [CrossRef]

180. Pfeifer, M.; Begerow, B.; Minne, H.W.; Nachtigall, D.; Hansen, C. Effects of a short-term vitamin D(3) and calcium supplementation on blood pressure and parathyroid hormone levels in elderly women. J. Clin. Endocrinol. Metab. 2001, 86, $1633-1637$. [CrossRef] [PubMed]

181. Kenny, A.M.; Biskup, B.; Robbins, B.; Marcella, G.; Burleson, J.A. Effects of vitamin D supplementation on strength, physical function, and health perception in older, community-dwelling men. J. Am. Geriatr. Soc. 2003, 51, 1762-1767. [CrossRef]

182. Grados, F.; Brazier, M.; Kamel, S.; Mathieu, M.; Hurtebize, N.; Maamer, M.; Garabédian, M.; Sebert, J.L.; Fardellone, P. Prediction of bone mass density variation by bone remodeling markers in postmenopausal women with vitamin D insufficiency treated with calcium and vitamin D supplementation. J. Clin. Endocrinol. Metab. 2003, 88, 5175-5179. [CrossRef] [PubMed]

183. Brazier, M.; Grados, F.; Kamel, S.; Mathieu, M.; Morel, A.; Maamer, M.; Sebert, J.L.; Fardellone, P. Clinical and laboratory safety of one year's use of a combination calcium + vitamin D tablet in ambulatory elderly women with vitamin D insufficiency: Results of a multicenter, randomized, double-blind, placebo-controlled study. Clin. Ther. 2005, 27, 1885-1893. [CrossRef] [PubMed]

184. Talwar, S.A.; Aloia, J.F.; Pollack, S.; Yeh, J.K. Dose response to vitamin D supplementation among postmenopausal African American women. Am. J. Clin. Nutr. 2007, 86, 1657-1662. [CrossRef]

185. Pittas, A.G.; Harris, S.S.; Stark, P.C.; Dawson-Hughes, B. The effects of calcium and vitamin D supplementation on blood glucose and markers of inflammation in nondiabetic adults. Diabetes Care 2007, 30, 980-986. [CrossRef] [PubMed]

186. Chel, V.; Wijnhoven, H.A.H.; Smit, J.H.; Ooms, M.; Lips, P. Efficacy of different doses and time intervals of oral vitamin D supplementation with or without calcium in elderly nursing home residents. Osteoporos. Int. 2008, 19, 663-671. [CrossRef] [PubMed]

187. Björkman, M.; Sorva, A.; Risteli, J.; Tilvis, R. Vitamin D supplementation has minor effects on parathyroid hormone and bone turnover markers in vitamin D-deficient bedridden older patients. Age Ageing 2008, 37, 25-31. [CrossRef]

188. Cashman, K.D.; Hill, T.R.; Lucey, A.J.; Taylor, N.; Seamans, K.M.; Muldowney, S.; FitzGerald, A.P.; Flynn, A.; Barnes, M.S.; Horigan, G.; et al. Estimation of the dietary requirement for vitamin D in healthy adults. Am. J. Clin. Nutr. 2008, 88, 1535-1542. [CrossRef]

189. Pfeifer, M.; Begerow, B.; Minne, H.W.; Suppan, K.; Fahrleitner-Pammer, A.; Dobnig, H. Effects of a long-term vitamin D and calcium supplementation on falls and parameters of muscle function in community-dwelling older individuals. Osteoporos. Int. 2009, 20, 315-322. [CrossRef]

190. Zittermann, A.; Frisch, S.; Berthold, H.K.; Götting, C.; Kuhn, J.; Kleesiek, K.; Stehle, P.; Koertke, H.; Koerfer, R. Vitamin D supplementation enhances the beneficial effects of weight loss on cardiovascular disease risk markers. Am. J. Clin. Nutr. 2009, 89, 1321-1327. [CrossRef]

191. Islam, M.Z.; Shamim, A.A.; Viljakainen, H.T.; Akhtaruzzaman, M.; Jehan, A.H.; Khan, H.U.; Al-Arif, F.A.; Lamberg-Allardt, C. Effect of vitamin D, calcium and multiple micronutrient supplementation on vitamin D and bone status in Bangladeshi premenopausal garment factory workers with hypovitaminosis D: A double-blinded, randomised, placebo-controlled 1-year intervention. Br. J. Nutr. 2010, 104, 241-247. [CrossRef]

192. Jorde, R.; Sneve, M.; Torjesen, P.; Figenschau, Y.; Hansen, J.B. Parameters of the thrombogram are associated with serum 25-hydroxyvitamin D levels at baseline, but not affected during supplementation with vitamin D. Thromb. Res. 2010, 125, e210-e213. [CrossRef]

193. Lips, P.; Binkley, N.; Pfeifer, M.; Recker, R.; Samanta, S.; Cohn, D.A.; Chandler, J.; Rosenberg, E.; Papanicolaou, D.A. Once-weekly dose of 8400 IU vitamin $\mathrm{D}(3)$ compared with placebo: Effects on neuromuscular function and tolerability in older adults with vitamin D insufficiency. Am. J. Clin. Nutr. 2010, 91, 985-991. [CrossRef]

194. Grimnes, G.; Figenschau, Y.; Almås, B.; Jorde, R. Vitamin D, insulin secretion, sensitivity, and lipids: Results from a case-control study and a randomized controlled trial using hyperglycemic clamp technique. Diabetes 2011, 60, 2748-2757. [CrossRef] [PubMed]

195. Sokol, S.I.; Srinivas, V.; Crandall, J.P.; Kim, M.; Tellides, G.; Lebastchi, A.; Yu, Y.; Gupta, A.K.; Alderman, M.H. The effects of vitamin D repletion on endothelial function and inflammation in patients with coronary artery disease. Vasc. Med. 2012, 17, 394-404. [CrossRef] [PubMed]

196. Ponda, M.P.; Dowd, K.; Finkielstein, D.; Holt, P.R.; Breslow, J.L. The short-term effects of vitamin D repletion on cholesterol: A randomized, placebo-controlled trial. Arterioscler. Thromb. Vasc. Biol. 2012, 32, 2510-2515. [CrossRef]

197. Harris, S.S.; Pittas, A.G.; Palermo, N.J. A randomized, placebo-controlled trial of vitamin D supplementation to improve glycaemia in overweight and obese African Americans. Diabetes Obes. Metab. 2012, 14, 789-794. [CrossRef]

198. Larsen, T.; Mose, F.H.; Bech, J.N.; Hansen, A.B.; Pedersen, E.B. Effect of cholecalciferol supplementation during winter months in patients with hypertension: A randomized, placebo-controlled trial. Am. J. Hypertens. 2012, 25, 1215-1222. [CrossRef]

199. Kjærgaard, M.; Waterloo, K.; Wang, C.E.A.; Almås, B.; Figenschau, Y.; Hutchinson, M.S.; Svartberg, J.; Jorde, R. Effect of vitamin D supplement on depression scores in people with low levels of serum 25-hydroxyvitamin D: Nested case-control study and randomised clinical trial. Br. J. Psychiatry 2012, 201, 360-368. [CrossRef]

200. Salehpour, A.; Hosseinpanah, F.; Shidfar, F.; Vafa, M.; Razaghi, M.; Dehghani, S.; Hoshiarrad, A.; Gohari, M. A 12-week doubleblind randomized clinical trial of vitamin $\mathrm{D}_{3}$ supplementation on body fat mass in healthy overweight and obese women. Nutr. J. 2012, 11, 78. [CrossRef] [PubMed] 
201. Goswami, R.; Vatsa, M.; Sreenivas, V.; Singh, U.; Gupta, N.; Lakshmy, R.; Aggarwal, S.; Ganapathy, A.; Joshi, P.; Bhatia, H. Skeletal muscle strength in young Asian Indian females after vitamin D and calcium supplementation: A double-blind randomized controlled clinical trial. J. Clin. Endocrinol. Metab. 2012, 97, 4709-4716. [CrossRef]

202. Suzuki, M.; Yoshioka, M.; Hashimoto, M.; Murakami, M.; Noya, M.; Takahashi, D.; Urashima, M. Randomized, double-blind, placebo-controlled trial of vitamin D supplementation in Parkinson disease. Am. J. Clin. Nutr. 2013, 97, 1004-1013. [CrossRef]

203. Jorde, R.; Bønaa, K.H. Calcium from dairy products, vitamin D intake, and blood pressure: The Tromsø study. Am. J. Clin. Nutr. 2000, 71, 1530-1535. [CrossRef]

204. Rudnicki, M.; Thode, J.; Jorgensen, T.; Heitmann, B.L.; Sorensen, O.H. Effects of age, sex, season and diet on serum ionized calcium, parathyroid hormone and vitamin D in a random population. J. Intern. Med. 1993, 234, 195-200. [CrossRef] [PubMed]

205. Khadilkar, A.; Mughal, M.Z.; Hanumante, N.; Sayyad, M.; Sanwalka, N.; Naik, S.; Fraser, W.D.; Joshi, A.; Khadilkar, V. Oral calcium supplementation reverses the biochemical pattern of parathyroid hormone resistance in underprivileged Indian toddlers. Arch. Dis. Child. 2009, 94, 932-937. [CrossRef]

206. Patel, P.; Zulf Mughal, M.; Patel, P.; Yagnik, B.; Kajale, N.; Mandlik, R.; Khadilkar, V.; Chiplonkar, S.A.; Phanse, S.; Patwardhan, V.; et al. Dietary calcium intake influences the relationship between serum 25-hydroxyvitamin D3 (25OHD) concentration and parathyroid hormone (PTH) concentration. Arch. Dis. Child. 2016, 101, 316-319. [CrossRef]

207. Gunther, C.W.; Legowski, P.A.; Lyle, R.M.; Weaver, C.M.; McCabe, L.D.; McCabe, G.P.; Peacock, M.; Teegarden, D. Parathyroid hormone is associated with decreased fat mass in young healthy women. Int. J. Obes. 2006, 30, 94-99. [CrossRef] [PubMed]

208. Ke, L.; Mason, R.S.; Mpofu, E.; Dibley, M.; Li, Y.; Brock, K.E. Vitamin D and parathyroid hormone status in a representative population living in Macau, China. J. Steroid Biochem. Mol. Biol. 2015, 148, 261-268. [CrossRef]

209. Muschitz, C.; Kocijan, R.; Haschka, J.; Zendeli, A.; Pirker, T.; Geiger, C.; Müller, A.; Tschinder, B.; Kocijan, A.; Marterer, C.; et al. The impact of vitamin D, calcium, protein supplementation, and physical exercise on bone metabolism after bariatric surgery: The BABS study. J. Bone Miner. Res. 2016, 31, 672-682. [CrossRef]

210. Bouassida, A.; Zalleg, D.; Zaouali Ajina, M.; Gharbi, N.; Duclos, M.; Richalet, J.P.; Tabka, Z. Parathyroid hormone concentrations during and after two periods of high intensity exercise with and without an intervening recovery period. Eur. J. Appl. Physiol. 2003, 88, 339-344. [CrossRef]

211. Brahm, H.; Piehl-Aulin, K.; Ljunghall, S. Bone metabolism during exercise and recovery: The influence of plasma volume and physical fitness. Calcif. Tissue Int. 1997, 61, 192-198. [CrossRef]

212. Dashtidehkordi, A.; Shahgholian, N.; Sadeghian, J. The effect of exercise during hemodialysis on serum levels of albumin, calcium, phosphorus and parathyroid hormone: A randomized clinical trial. Res. Square 2021, 1-18. [CrossRef]

213. Falk, B.; Haddad, F.; Klentrou, P.; Ward, W.; Kish, K.; Mezil, Y.; Radom-Aizik, S. Differential sclerostin and parathyroid hormone response to exercise in boys and men. Osteoporos. Int. 2016, 27, 1245-1249. [CrossRef]

214. Kohrt, W.M.; Wherry, S.J.; Wolfe, P.; Sherk, V.D.; Wellington, T.; Swanson, C.M.; Weaver, C.M.; Boxer, R.S. Maintenance of serum ionized calcium during exercise attenuates parathyroid hormone and bone resorption responses. J. Bone Miner. Res. 2018, 33, 1326-1334. [CrossRef]

215. Kohrt, W.M.; Wolfe, P.; Sherk, V.D.; Wherry, S.J.; Wellington, T.; Melanson, E.L.; Swanson, C.M.; Weaver, C.M.; Boxer, R.S. Dermal calcium loss is not the primary determinant of parathyroid hormone secretion during exercise. Med. Sci. Sport. Exerc. 2019, 51, 2117-2124. [CrossRef]

216. Ljunghall, S.; Joborn, H.; Roxin, L.-E.; Rastad, J.; Wide, L.; Åkerström, G. Prolonged low-intensity exercise raises the serum parathyroid hormone levels. Clin. Endocrinol. 1986, 25, 535-542. [CrossRef]

217. Moreira, L.D.F.; Fronza, F.C.A.O.; Dos Santos, R.N.; Zach, P.L.; Kunii, I.S.; Hayashi, L.F.; Teixeira, L.R.; Kruel, L.F.M.; Castro, M.L. The benefits of a high-intensity aquatic exercise program (HydrOS) for bone metabolism and bone mass of postmenopausal women. J. Bone Miner. Metab. 2014, 32, 411-419. [CrossRef]

218. Maïmoun, L.; Simar, D.; Malatesta, D.; Caillaud, C.; Peruchon, E.; Couret, I.; Rossi, M.; Mariano-Goulart, D. Response of bone metabolism related hormones to a single session of strenuous exercise in active elderly subjects. Br. J. Sport. Med. 2005, 39, 497-502. [CrossRef]

219. Maïmoun, L.; Manetta, J.; Couret, I.; Dupuy, A.M.; Mariano-Goulart, D.; Micallef, J.P.; Peruchon, E.; Rossi, M. The intensity level of physical exercise and the bone metabolism response. Int. J. Sport. Med. 2006, 27, 105-111. [CrossRef]

220. Mathis, S.L.; Pivovarova, A.I.; Hicks, S.M.; Alrefai, H.; MacGregor, G.G. Calcium loss in sweat does not stimulate PTH release: A study of Bikram hot yoga. Complement. Ther. Med. 2020, 51, 102417. [CrossRef]

221. Salvesen, H.; Johansson, A.G.; Foxdal, P.; Wide, L.; Piehl-Aulin, K.; Ljunghall, S. Intact serum parathyroid hormone levels increase during running exercise in well-trained men. Calcif. Tissue Int. 1994, 54, 256-261. [CrossRef]

222. Scott, J.P.R.; Sale, C.; Greeves, J.P.; Casey, A.; Dutton, J.; Fraser, W.D. The role of exercise intensity in the bone metabolic response to an acute bout of weight-bearing exercise. J. Appl. Physiol. 2011, 110, 423-432. [CrossRef]

223. Scott, J.P.R.; Sale, C.; Greeves, J.P.; Casey, A.; Dutton, J.; Fraser, W.D. Treadmill running reduces parathyroid hormone concentrations during recovery compared with a nonexercising control group. J. Clin. Endocrinol. Metab. 2014, 99, 1774-1782. [CrossRef]

224. Sherk, V.D.; Wherry, S.J.; Barry, D.W.; Shea, K.L.; Wolfe, P.; Kohrt, W.M. Calcium supplementation attenuates disruptions in calcium homeostasis during exercise. Med. Sci. Sport. Exerc. 2017, 49, 1437. [CrossRef]

225. Takada, H.; Washino, K.; Hanai, T.; Iwata, H. Response of parathyroid hormone to exercise and bone mineral density in adolescent female athletes. Environ. Health Prev. Med. 1998, 2, 161-166. [CrossRef] 
226. Thorsen, K.; Kristoffersson, A.; Hultdin, J.; Lorentzon, R. Effects of moderate endurance exercise on calcium, parathyroid hormone, and markers of bone metabolism in young women. Calcif. Tissue Int. 1997, 60, 16-20. [CrossRef]

227. Townsend, R.; Elliott-Sale, K.J.; Pinto, A.J.; Thomas, C.; Scott, J.P.R.; Currell, K.; Fraser, W.D.; Sale, C. Parathyroid hormone secretion is controlled by both ionized calcium and phosphate during exercise and recovery in men. J. Clin. Endocrinol. Metab. 2016, 101, 3231-3239. [CrossRef]

228. Ljunghall, S.; Joborn, H.; Roxin, L.E.; Skarfors, E.T.; Wide, L.E.; Lithell, H.O. Increase in serum parathyroid hormone levels after prolonged physical exercise. Med. Sci. Sport. Exerc. 1988, 20, 122-125. [CrossRef] [PubMed]

229. Diaz-Castro, J.; Mira-Rufino, P.J.; Moreno-Fernandez, J.; Chirosa, I.; Chirosa, J.L.; Guisado, R.; Ochoa, J.J. Ubiquinol supplementation modulates energy metabolism and bone turnover during high intensity exercise. Food Funct. 2020, 11, 7523-7531. [CrossRef]

230. Malandish, A.; Tartibian, B.; Sheikhlou, Z.; Afsargharehbagh, R.; Rahmati, M. The effects of short-term moderate intensity aerobic exercise and long-term detraining on electrocardiogram indices and cardiac biomarkers in postmenopausal women. J. Electrocardiol. 2020, 60, 15-22. [CrossRef]

231. Tsai, K.S.; Lin, J.C.; Chen, C.K.; Cheng, W.C.; Yang, C.H. Effect of exercise and exogenous glucocorticoid on serum level of intact parathyroid hormone. Int. J. Sport. Med. 1997, 18, 583-587. [CrossRef]

232. Vora, N.M.; Kukreja, S.C.; York, P.A.J.; Bowser, E.N.; Hargis, G.K.; Williams, G.A. Effect of exercise on serum calcium and parathyroid hormone. J. Clin. Endocrinol. Metab. 1983, 57, 1067-1069. [CrossRef]

233. D'Herbomez, M.; Caron, P.; Bauters, C.; Do Cao, C.; Schlienger, J.L.; Sapin, R.; Baldet, L.; Carnaille, B.; Wémeau, J.L. Reference range of serum calcitonin levels in humans: Influence of calcitonin assays, sex, age, and cigarette smoking. Eur. J. Endocrinol. 2007, 157, 749-755. [CrossRef]

234. Gobba, N.A.E.K.; Hussein Ali, A.; El Sharawy, D.E.; Hussein, M.A. The potential hazardous effect of exposure to iron dust in Egyptian smoking and nonsmoking welders. Arch. Environ. Occup. Health 2017, 73, 189-202. [CrossRef]

235. Cvek, M.; Punda, A.; Brekalo, M.; PLoSnić, M.; Barić, A.; Kaličanin, D.; Brčić, L.; Vuletić, M.; Gunjača, I.; Torlak Lovrić, V.; et al. Presence or severity of Hashimoto's thyroiditis does not influence basal calcitonin levels: Observations from CROHT biobank. J. Endocrinol. Investig. 2021, 1-9. [CrossRef]

236. Sabia, R.; Wagner, M.; Susa, K.; Lemke, J.; Rothermund, L.; Henne-Bruns, D.; Hillenbrand, A. Calcitonin concentrations in patients with chronic kidney disease on hemodialysis in reference to parathyroidectomy. BMC Res. Notes 2019, 12, 1-5. [CrossRef]

237. Pilat-Marcinkiewicz, B.; Brzóska, M.; Moniuszko-Jakoniuk, J. Thyroid and parathyroid function and structure in male rats chronically exposed to cadmium. Pol. J. Environ. Stud. 2008, 17, 113-120.

238. Nogawa, K.; Kobayashi, E.; Yamada, Y.; Honda, R.; Kido, T.; Tsuritani, I.; Ishizaki, M. Parathyroid hormone concentration in the serum of people with cadmium-induced renal damage. Int. Arch. Occup. Environ. Health 1984, 54, 187-193. [CrossRef] [PubMed]

239. Tsuritani, I.; Honda, R.; Lshizaki, M.; Yamada, Y.; Kido, T.; Nogawa, K. Impairment of vitamin D metabolism due to environmental cadmium exposure, and possible relevance to sex-related differences in vulnerability to the bone damage. J. Toxicol. Environ. Health 1992, 37, 519-533. [CrossRef]

240. Jarup, L.; Persson, B.; Elinder, C.G. Decreased glomerular filtration rate in solderers exposed to cadmium. Occup. Environ. Med. 1995, 52, 818. [CrossRef]

241. Nogawa, K.; Tsuritani, I.; Kido, T.; Honda, R.; Yamada, Y.; Ishizaki, M. Mechanism for bone disease found in inhabitants environmentally exposed to cadmium: Decreased serum 1 alpha, 25-dihydroxyvitamin D level. Int. Arch. Occup. Environ. Health 1987, 59, 21-30. [CrossRef] [PubMed]

242. Malin Igra, A.; Vahter, M.; Raqib, R.; Kippler, M. Early-life cadmium exposure and bone-related biomarkers: A longitudinal study in children. Environ. Health Perspect. 2019, 127, 37003. [CrossRef] [PubMed]

243. Brzóska, M.M.; Moniuszko-Jakoniuk, J. Bone metabolism of male rats chronically exposed to cadmium. Toxicol. Appl. Pharmacol. 2005, 207, 195-211. [CrossRef]

244. Ahmed, S.; Rekha, R.S.; Ahsan, K.B.; Doi, M.; Grandér, M.; Roy, A.K.; Ekström, E.C.; Wagatsuma, Y.; Vahter, M.; Raqib, R. Arsenic exposure affects plasma insulin-like growth factor 1 (IGF-1) in children in rural Bangladesh. PLoS ONE 2013, 8, e81530. [CrossRef]

245. Mazumdar, I.; Goswami, K.; Ali, M.S. Status of serum calcium, vitamin D and parathyroid hormone and hematological indices among lead exposed jewelry workers in Dhaka, Bangladesh. Indian J. Clin. Biochem. 2017, 32, 110. [CrossRef]

246. McDiarmid, M.A.; Engelhardt, S.M.; Dorsey, C.D.; Oliver, M.; Gucer, P.; Gaitens, J.M.; Kane, R.; Cernich, A.; Kaup, B.; Hoover, D.; et al. Longitudinal health surveillance in a cohort of Gulf War veterans 18 years after first exposure to depleted uranium. J. Toxicol. Environ. Health A 2011, 74, 678-691. [CrossRef]

247. Yuan, G.; Lu, H.; Yin, Z.; Dai, S.; Jia, R.; Xu, J.; Song, X.; Li, L. Effects of mixed subchronic lead acetate and cadmium chloride on bone metabolism in rats. Int. J. Clin. Exp. Med. 2014, 7, 1378.

248. Zhu, M.; Zhou, W.; Bai, L.; Li, H.; Wang, L.; Zou, X. Dietary cadmium chloride supplementation impairs renal function and bone metabolism of laying hens. Animals 2019, 9, 998. [CrossRef]

249. Suzuki, N.; Yamamoto, M.; Watanabe, K.; Kambegawa, A.; Hattori, A. Both mercury and cadmium directly influence calcium homeostasis resulting from the suppression of scale bone cells: The scale is a good model for the evaluation of heavy metals in bone metabolism. J. Bone Miner. Metab. 2004, 22, 439-446. [CrossRef]

250. Guo, Y.L.; Lin, C.J.; Yao, W.J.; Ryan, J.J.; Hsu, C.C. Musculoskeletal changes in children prenatally exposed to polychlorinated biphenyls and related compounds (Yu-Cheng children). J. Toxicol. Environ. Health 1994, 41, 83-93. [CrossRef] [PubMed] 
251. Di Nisio, A.; Rocca, M.S.; De Toni, L.; Sabovic, I.; Guidolin, D.; Dall'Acqua, S.; Acquasaliente, L.; De Filippis, V.; Plebani, M.; Foresta, C. Endocrine disruption of vitamin D activity by perfluoro-octanoic acid (PFOA). Sci. Rep. 2020, 10, 1-12. [CrossRef] [PubMed]

252. Koroglu, B.K.; Ersoy, I.H.; Koroglu, M.; Balkarli, A.; Ersoy, S.; Varol, S.; Tamer, M.N. Serum parathyroid hormone levels in chronic endemic fluorosis. Biol. Trace Elem. Res. 2011, 143, 79-86. [CrossRef] [PubMed]

253. Skórka-Majewicz, M.; Goschorska, M.; Żwierełło, W.; Baranowska-Bosiacka, I.; Styburski, D.; Kapczuk, P.; Gutowska, I. Effect of fluoride on endocrine tissues and their secretory functions-Review. Chemosphere 2020, 260, 127565. [CrossRef] [PubMed]

254. Ibrahim, M.A.A.; Elkaliny, H.H.; Abd-Elsalam, M.M. Lycopene ameliorates the effect of Aroclor 1254 on morphology, proliferation, and angiogenesis of the thyroid gland in rat. Toxicology 2021, 452, 152722. [CrossRef]

255. Gore, A.C.; Chappell, V.A.; Fenton, S.E.; Flaws, J.A.; Nadal, A.; Prins, G.S.; Toppari, J.; Zoeller, R.T. EDC-2: The Endocrine Society's second scientific statement on endocrine-disrupting chemicals. Endocr. Rev. 2015, 36, 1-150. [CrossRef]

256. Zeng, Q.b.; Xu, Y.y.; Yu, X.; Yang, J.; Hong, F.; Zhang, A. hua Arsenic may be involved in fluoride-induced bone toxicity through PTH/PKA/AP1 signaling pathway. Environ. Toxicol. Pharmacol. 2014, 37, 228-233. [CrossRef]

257. Engström, A.; Skerving, S.; Lidfeldt, J.; Burgaz, A.; Lundh, T.; Samsioe, G.; Vahter, M.; Åkesson, A. Cadmium-induced bone effect is not mediated via low serum 1,25-dihydroxy vitamin D. Environ. Res. 2009, 109, 188-192. [CrossRef] [PubMed]

258. Ibrahim, K.S.; Beshir, S.; Shahy, E.M.; Shaheen, W.A. Effect of occupational cadmium exposure on parathyroid gland. Open Access Maced. J. Med. Sci. 2016, 4, 302. [CrossRef] [PubMed]

259. Kido, T.; Honda, R.; Tsuritani, I.; Ishizaki, M.; Yamada, Y.; Nogawa, K.; Nakagawa, H.; Dohi, Y. Assessment of cadmium-induced osteopenia by measurement of serum bone Gla protein, parathyroid hormone, and 1 alpha,25-dihydroxyvitamin D. J. Appl. Toxicol. 1991, 11, 161-166. [CrossRef]

260. Lee, C.C.; Weng, C.H.; Huang, W.H.; Yen, T.H.; Lin, J.L.; Lin, D.T.; Chen, K.H.; Hsu, C.W. Association between blood cadmium levels and mortality in peritoneal dialysis. Medicine 2016, 95, e3717. [CrossRef]

261. Ghosh-Narang, J.; Jones, T.M.; Menke, A.; Todd, A.C.; Muntner, P.; Batuman, V. Parathyroid hormone status does not influence blood and bone lead levels in dialysis patients. Am. J. Med. Sci. 2007, 334, 415-420. [CrossRef]

262. Kristal-Boneh, E.; Froom, P.; Yerushalmi, N.; Harari, G.; Ribak, J. Calcitropic hormones and occupational lead exposure. Am. J. Epidemiol. 1998, 147, 458-463. [CrossRef] [PubMed]

263. Lin, J.L.; Lin-Tan, D.T.; Chen, K.H.; Hsu, C.W.; Yen, T.H.; Huang, W.H.; Huang, Y.L. Blood lead levels association with 18-month all-cause mortality in patients with chronic peritoneal dialysis. Nephrol. Dial. Transplant. 2010, 25, 1627-1633. [CrossRef] [PubMed]

264. Colleoni, N.; Arrigo, G.; Gandini, E.; Corigliano, C.; D'Amico, G. Blood lead in hemodialysis patients. Am. J. Nephrol. 1993, 13, 198-202. [CrossRef] [PubMed]

265. Osterloh, J.D. Observations on the effect of parathyroid hormone on environmental blood lead concentrations in humans. Environ. Res. 1991, 54, 8-16. [CrossRef]

266. Potula, V.; Henderson, A.; Kaye, W. Calcitropic hormones, bone turnover, and lead exposure among female smelter workers. Arch. Environ. Occup. Health 2005, 60, 195-204. [CrossRef]

267. Pouresmaeil, R.; Razeghi, E.; Ahmadi, F. Correlation of serum lead levels with inflammation, nutritional status, and clinical complications in hemodialysis patients. Ren. Fail. 2012, 34, 1114-1117. [CrossRef]

268. McDiarmid, M.A.; Engelhardt, S.M.; Dorsey, C.D.; Oliver, M.; Gucer, P.; Wilson, P.D.; Kane, R.; Cernich, A.; Kaup, B.; Anderson, L.; et al. Surveillance results of depleted uranium-exposed Gulf War I veterans: Sixteen years of follow-up. J. Toxicol. Environ. Health A 2009, 72, 14-29. [CrossRef] [PubMed]

269. Thippeswamy, H.M.; Devananda, D.; Nanditha Kumar, M.; Wormald, M.M.; Prashanth, S.N. The association of fluoride in drinking water with serum calcium, vitamin D and parathyroid hormone in pregnant women and newborn infants. Eur. J. Clin. Nutr. 2020, 75, 151-159. [CrossRef] [PubMed]

270. Harari, F.; Åkesson, A.; Casimiro, E.; Lu, Y.; Vahter, M. Exposure to lithium through drinking water and calcium homeostasis during pregnancy: A longitudinal study. Environ. Res. 2016, 147, 1-7. [CrossRef] 